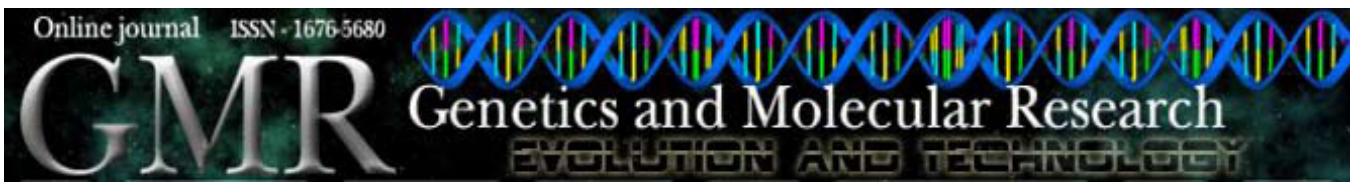

\title{
Population genetic structure of the migratory rice leaf roller, Cnaphalocrocis medinalis (Lepidoptera: Pyralidae), inferred from the mitochondrial A+T-rich region and nuclear ITS2 sequences
}

\author{
X. Wan' ${ }^{1}$ J. Li ${ }^{2}$, M.J. Kim¹, T.H. Kang ${ }^{3}$, B.R. Jin ${ }^{4}$ and I. Kim ${ }^{1}$ \\ ${ }^{1}$ College of Agriculture and Life Sciences, Chonnam National University, \\ Gwangju, Republic of Korea \\ ${ }^{2}$ College of Plant Science and Technology, Huazhong Agricultural University, \\ Wuhan, Hubei, China \\ ${ }^{3}$ Department of Agricultural Biology, National Academy of Agricultural Science, \\ Suwon, Republic of Korea \\ ${ }^{4}$ College of Natural Resources and Life Science, Dong-A University, Busan, \\ Republic of Korea \\ Corresponding author: I. Kim \\ E-mail: ikkim81@chonnam.ac.kr
}

Genet. Mol. Res. 10 (1): 273-294 (2011)

Received September 9, 2010

Accepted November 10, 2010

Published February 22, 2011

DOI 10.4238/vol10-1gmr1005

ABSTRACT. The population genetics of the migratory rice leaf roller, Cnaphalocrocis medinalis (Lepidoptera: Pyralidae), was characterized using the maternally inherited mitochondrial $\mathrm{A}+\mathrm{T}$ rich region and bi-parentally inherited nuclear internal transcribed spacer 2 (ITS2). One hundred and eighty-seven specimens of the rice leaf roller collected from 13 Korean and Chinese localities revealed $94 \mathrm{~A}+\mathrm{T}$-rich region haplotypes, ranging in sequence length from 339 to $348 \mathrm{bp}$ and 129 ITS2 sequence types, ranging from 444 to $450 \mathrm{bp}$, with maximum sequence divergences of 4.55 and $4.43 \%$, respectively. The finding of almost no significant $F_{\text {ST, }}$ even among Chinese and Korean localities, except for one 
Chinese island population (ITS2 only), and the finding of genetic variance principally at the within-population level indicate the genetic structure characteristics of a migratory insect that is well connected among populations due to high gene flow. Detection of significant $F_{\text {ST }}$ estimates of one offshore island population in China (Haikou) compared to most others only by ITS2 rather than by the mitochondrial $\mathrm{A}+\mathrm{T}$-rich region, as well as the somewhat higher degree of genetic differentiation seen on ITS2, suggest the importance of female dispersal. Structural analysis of the A+T-rich region revealed a poly-T stretch (10-16 bp), a microsatellite-like AT repeat (10-14 repeats), and a 5-bp long-motif “ATTTA". The typical 5-bp long conserved motif sequence (ATAGA) previously detected in other lepidopterans was found to be ATAG in the C. medinalis $\mathrm{A}+\mathrm{T}$-rich region.

Key words: Cnaphalocrocis medinalis; A+T-rich region; ITS2; Population genetics; Genetic diversity; Gene flow

\section{INTRODUCTION}

Genetic variability, the source of evolution upon which the maintenance and persistence of an organism depends, is affected by a variety of factors. These include mutation, population size, and gene flow (Amos and Harwood, 1998). Population size is of primary importance, as a reduction in population size may result in a loss of diversity due to genetic drift. This force, on the other hand, may be compensated by dispersal, via which individual populations can be interconnected to form integrated metapopulations (Hanski, 1996). On the other hand, the genetic distance among separated populations may be generated by differential selection forces specific to each population, habitat fragmentation, geographic isolation, limited gene flow, and so on (Nei and Feldman, 1972; Zhang et al., 2009). In particular, a species that occupies ephemeral habitats is likely to face extinction if it is incapable of dispersal, whereas dispersal is not expected to play as large a role in stable habitats (Denno et al., 1996).

The rice leaf roller, Cnaphalocrocis medinalis (Lepidoptera: Pyralidae), feeds on a broad range of graminaceous crops, but primarily on rice, and it is widely distributed throughout South and East Asia (Kisimoto, 1984). A substantial amount of research has been conducted on the dispersal of this species in Asia (Miyahara, 1981; Wada et al., 1988; Riley et al., 1995). Although an understanding of the genetic population structures of insect pests may provide us with important biological information, no such studies have yet been conducted on C. medinalis. Available examples include members of the same superfamily, Pyraloidea, including the European corn borer, Ostrinia nubilalis and the rice stem borer Chilo suppressalis.

It has been suggested that population genetics can be illustrated more accurately than was previously possible by analyzing both mitochondrial and nuclear markers (Ballard and Whitlock, 2004). This is because each molecule evidences its own evolutionary characteristics that allow for inferences of different aspects of population history. For example, mitochondrial DNA has an 
effective population size one-quarter that of nuclear DNA, resulting in stronger effects of genetic drift, more rapid fixation, and stronger population subdivision (Birky Jr. et al., 1983). Further, inference of population structure on the basis of a single molecular marker could be either exaggerated or unrecognizable whenever dispersal is gender-biased (Birky Jr. et al., 1983).

We evaluated the population genetics of the migratory rice leaf roller, C. medinalis, for the first time, using the mitochondrial $\mathrm{A}+\mathrm{T}$-rich region and nuclear internal transcribed spacer 2 (ITS2). Each molecular sequence is widely regarded as a powerful tool for the study of population genetics and phylogenetic reconstruction of closely related taxa (Bravo et al., 2008; Depaquit et al., 2008). Considering that each molecule has its own evolutionary characteristics, using both molecules is expected to provide greater insight into more diverse aspects of population genetics than was previously possible (Ballard and Whitlock, 2004). In particular, by employing two molecular sequences we addressed the following questions: First, what is the magnitude of genetic diversity and gene flow among C. medinalis populations? Second, how is the gene flow reflected in these two molecules? Third, can any bias in dispersal be detected between the two genders in $C$. medinalis?

\section{MATERIAL AND METHODS}

\section{Sampling}

Larvae and adults of $C$. medinalis were collected from rice plants in 13 localities in Korea and China from 2008 to 2009 (Table 1; Figure 1). Each larva or adult specimen was collected at least one meter from the neighboring one, in order to cover a wide area of each collecting locality.

\section{DNA extraction, primers, and amplification}

Total DNA was extracted with a DNA purification kit (Promega, USA). In order to amplify the entirety of the mitochondrial A+T-rich region, a pair of primers was designed on the basis of the full-length mitogenome sequences of lepidopterans (Yukuhiro et al., 2002; Cameron and Whiting, 2008). The forward primer (ATF, 5'-AATAATAGGGTATCTAATCCTAG-3') and reverse primer (ATR, 5'-AATTTATCCTATCAGAATAATCC-3') are located between the 12S rRNA and tRNA ${ }^{\text {Ile }}$ genes, respectively. The polymerase chain reactions (PCR) were conducted as follows: denaturation for $7 \mathrm{~min}$ at $94^{\circ} \mathrm{C}$, followed by 35 cycles of 1 min denaturation at $94^{\circ} \mathrm{C}, 1$ min annealing at $55.6^{\circ} \mathrm{C}$, and $1 \mathrm{~min}$ extension at $72^{\circ} \mathrm{C}$, and a final extension for $7 \mathrm{~min}$ at $72^{\circ} \mathrm{C}$. In order to amplify the ITS2, the primers NG02955, 5'-ATGAACATCGACATTTCGAACGCACA T-3' and AB052895, 5'-TTCTTTTCCTCCGCTTAGTAATATGCTTAA-3' (Ji et al., 2003) were used. After an initial 4-min denaturation step at $94^{\circ} \mathrm{C}$, a 35 -cycle amplification $\left(95^{\circ} \mathrm{C}\right.$ for $40 \mathrm{~s}$, $64^{\circ} \mathrm{C}$ for $20 \mathrm{~s}$, and $72^{\circ} \mathrm{C}$ for $40 \mathrm{~s}$ ) was run. The final extension step was conducted for $2 \mathrm{~min}$ and $40 \mathrm{~s}$ at $72^{\circ} \mathrm{C}$. PCR amplification was conducted using AccuPower ${ }^{\circledR}$ PCR PreMix (Bioneer, Korea) in a Biometra thermal cycler (model T-gradient Thermoblock, Germany).

The amplicons of the A+T-rich region and ITS2 were subsequently purified and cloned into pGEM-T Easy vector (Promega), and the plasmid DNA from one clone per individual was isolated with a Plasmid Miniprep Kit (Dyne Bio Inc., Korea). Both strands of the plasmid were sequenced with an ABI PRISM ${ }^{\mathrm{TM}} 3100$ Genetic Analyzer (PE Applied Biosystems, USA). 


\begin{tabular}{|c|c|c|c|c|c|c|c|c|c|c|c|}
\hline \multirow{2}{*}{$\begin{array}{l}\text { Collecting locality } \\
\text { (sample size, } \\
\text { collecting date) }\end{array}$} & \multirow{2}{*}{$\begin{array}{l}\text { Animal } \\
\text { number }\end{array}$} & \multicolumn{5}{|c|}{$\mathrm{A}+\mathrm{T}$-rich region } & \multicolumn{5}{|c|}{ ITS2 } \\
\hline & & $\begin{array}{c}\text { Haplotype } \\
\text { I* }\end{array}$ & $\begin{array}{l}\text { AT } \\
\text { content } \\
(\%) \\
\end{array}$ & $\begin{array}{l}\text { Sequence } \\
\text { size (bp) }\end{array}$ & $\begin{array}{c}\text { GenBank } \\
\text { accession } \\
\text { No. }\end{array}$ & $\begin{array}{c}\text { Haplotype } \\
\text { II** }\end{array}$ & $\begin{array}{l}\text { Sequence } \\
\text { type I* }\end{array}$ & $\begin{array}{c}\mathrm{GC} \\
\text { content } \\
(\%) \\
\end{array}$ & $\begin{array}{l}\text { Sequence } \\
\text { size } \\
(\mathrm{bp})\end{array}$ & $\begin{array}{c}\text { GenBank } \\
\text { accession } \\
\text { No. }\end{array}$ & $\begin{array}{l}\text { Sequence } \\
\text { type II** }\end{array}$ \\
\hline \multirow{15}{*}{$\begin{array}{l}\text { 1. Haikou, } \\
\text { Hainan Island, } \\
\text { China } \\
(15 ; 2009.08 .15)\end{array}$} & CM3241 & СMAT01 & 96.0 & 344 & HM755071 & СMAT01 & CMITS013 & 47.5 & 448 & HM755258 & CMITS009 \\
\hline & CM3242 & CMAT06 & 95.9 & 342 & HM755072 & СMAT01 & CMITS014 & 47.6 & 445 & HM755259 & CMITS010 \\
\hline & CM3243 & CMAT11 & 95.9 & 345 & HM755073 & CMAT06 & CMITS005 & 48.1 & 445 & HM755260 & CMITS004 \\
\hline & CM3244 & СMAT01 & 96.0 & 344 & HM755074 & СMAT01 & CMITS015 & 47.2 & 447 & HM755261 & CMITS011 \\
\hline & CM3245 & CMAT01 & 96.0 & 344 & HM755075 & CMAT01 & CMITS016 & 48.3 & 445 & HM755262 & CMITS012 \\
\hline & CM3246 & СMAT01 & 96.0 & 344 & HM755076 & CMAT01 & CMITS017 & 48.3 & 445 & HM755263 & CMITS013 \\
\hline & CM3247 & CMAT12 & 95.6 & 343 & HM755077 & CMAT07 & CMITS018 & 47.4 & 445 & HM755264 & CMITS014 \\
\hline & CM3248 & CMAT13 & 95.6 & 345 & HM755078 & СМАТ08 & CMITS019 & 47.7 & 449 & HM755265 & CMITS015 \\
\hline & CM3249 & CMAT05 & 95.9 & 345 & HM755079 & СMAT01 & CMITS020 & 48.3 & 445 & HM755266 & CMITS016 \\
\hline & CM 3250 & CMAT14 & 95.6 & 339 & HM755080 & СMAT09 & CMITS005 & 48.1 & 445 & HM755267 & CMITS004 \\
\hline & CM3251 & CMAT01 & 96.0 & 344 & HM755081 & СMAT01 & CMITS021 & 47.2 & 445 & HM755268 & CMITS017 \\
\hline & CM 3252 & СMAT04 & 95.9 & 345 & HM755082 & СMAT01 & CMITS022 & 47.5 & 446 & HM755269 & CMITS018 \\
\hline & CM3253 & CMAT01 & 96.0 & 344 & HM755083 & СMAT01 & CMITS005 & 48.1 & 445 & HM755270 & CMITS004 \\
\hline & CM3254 & CMAT15 & 95.9 & 344 & HM755084 & CMAT10 & CMITS023 & 48.1 & 445 & HM755271 & CMITS019 \\
\hline & CM3255 & СMAT01 & 96.0 & 344 & HM755085 & СMAT01 & CMITS024 & 48.3 & 445 & HM755272 & CMITS020 \\
\hline \multirow{15}{*}{$\begin{array}{l}\text { 2. Tonghai, } \\
\text { Yunnan Province, } \\
\text { China } \\
(15 ; 2009.07 .16)\end{array}$} & CM2903 & СMAT04 & 95.9 & 345 & HM755086 & CMAT01 & CMITS001 & 47.7 & 445 & HM755273 & CMITS001 \\
\hline & CM2904 & CMAT16 & 95.6 & 345 & HM755087 & CMAT11 & CMITS025 & 47.9 & 445 & HM755274 & CMITS021 \\
\hline & CM2905 & CMAT02 & 95.9 & 343 & HM755088 & CMAT01 & CMITS026 & 47.7 & 445 & HM755275 & CMITS022 \\
\hline & CM2906 & СMAT02 & 95.9 & 343 & HM755089 & CMAT01 & CMITS001 & 47.7 & 445 & HM755276 & CMITS001 \\
\hline & CM2907 & CMAT01 & 96.0 & 344 & HM755090 & CMAT01 & CMITS027 & 48.1 & 445 & HM755277 & CMITS023 \\
\hline & CM2908 & CMAT17 & 95.7 & 344 & HM755091 & CMAT12 & CMITS008 & 47.5 & 447 & HM755278 & CMITS001 \\
\hline & CM2909 & CMAT03 & 95.9 & 342 & HM755092 & CMAT01 & CMITS001 & 47.7 & 445 & HM755279 & CMITS001 \\
\hline & CM2910 & СMAT02 & 95.9 & 343 & HM755093 & СMAT01 & CMITS009 & 47.3 & 448 & HM755280 & CMITS001 \\
\hline & CM2911 & CMAT05 & 95.9 & 345 & HM755094 & CMAT01 & CMITS028 & 47.9 & 445 & HM755281 & CMITS024 \\
\hline & CM2912 & CMAT18 & 95.6 & 342 & HM755095 & CMAT03 & CMITS029 & 47.7 & 448 & HM755282 & CMITS025 \\
\hline & CM2913 & СMAT03 & 95.9 & 342 & HM755096 & CMAT01 & CMITS030 & 47.4 & 445 & HM755283 & CMITS026 \\
\hline & CM2914 & CMAT19 & 95.7 & 344 & HM755097 & CMAT13 & CMITS001 & 47.7 & 445 & HM755284 & CMITS001 \\
\hline & CM2915 & СМАT03 & 95.9 & 342 & HM755098 & СMAT01 & CMITS001 & 47.7 & 445 & HM755285 & CMITS001 \\
\hline & CM2916 & CMAT01 & 96.0 & 344 & HM755099 & CMAT01 & CMITS031 & 48.3 & 445 & HM755286 & CMITS027 \\
\hline & CM2917 & CMAT06 & 95.9 & & HM755100 & CMAT01 & CMITS032 & 47.5 & 448 & HM755287 & CMITS028 \\
\hline \multirow{7}{*}{$\begin{array}{l}\text { 3. Guangzhou, } \\
\text { Guangdong } \\
\text { Province, China } \\
(7 ; 2009.07 .17)\end{array}$} & CM2924 & CMAT02 & 95.9 & 343 & HM755101 & CMAT01 & CMITS002 & 47.9 & 445 & HM755288 & CMITS002 \\
\hline & CM2925 & СMAT01 & 96.0 & 344 & HM755102 & СMAT01 & CMITS033 & 47.4 & 445 & HM755289 & CMITS029 \\
\hline & CM2926 & СMAT01 & 96.0 & 344 & HM755103 & CMAT01 & CMITS002 & 47.9 & 445 & HM755290 & CMITS002 \\
\hline & CM2927 & CMAT02 & 95.9 & 343 & HM755104 & CMAT01 & CMITS001 & 47.7 & 445 & HM755291 & CMITS001 \\
\hline & CM2928 & CMAT06 & 95.9 & 342 & HM755105 & CMAT01 & CMITS001 & 47.7 & 445 & HM755292 & CMITS001 \\
\hline & CM2929 & CMAT01 & 96.0 & 344 & HM755106 & CMAT01 & CMITS006 & 47.8 & 445 & HM755293 & CMITS005 \\
\hline & CM2930 & CMAT01 & 96.0 & 344 & HM755107 & СMAT01 & CMITS006 & 47.8 & 445 & HM755294 & CMITS005 \\
\hline \multirow{15}{*}{$\begin{array}{l}\text { 4. Changsha, } \\
\text { Hunan Province, } \\
\text { China } \\
(15 ; 2009.07 .15)\end{array}$} & CM2931 & СMAT20 & 95.9 & 343 & HM755108 & CMAT14 & CMITS034 & 47.6 & 448 & HM755295 & CMITS030 \\
\hline & CM2932 & CMAT21 & 95.6 & 343 & HM755109 & CMAT15 & CMITS001 & 47.7 & 445 & HM755296 & CMITS001 \\
\hline & CM2933 & CMAT22 & 95.7 & 343 & HM755110 & СMAT03 & CMITS035 & 47.8 & 445 & HM755297 & CMITS031 \\
\hline & CM2934 & CMAT01 & 96.0 & 344 & HM755111 & СMAT01 & CMITS036 & 47.9 & 445 & HM755298 & CMITS032 \\
\hline & CM2935 & CMAT23 & 95.4 & 344 & HM755112 & CMAT16 & CMITS037 & 47.9 & 445 & HM755299 & CMITS033 \\
\hline & CM2936 & CMAT24 & 95.9 & 339 & & CMAT01 & CMITS001 & 47.7 & 44 & HM755300 & CMITS001 \\
\hline & CM2937 & CMAT01 & 96.0 & 344 & HM755114 & & & 47.1 & 44 & HM755301 & CMITS034 \\
\hline & CM2938 & CMAT25 & 95.6 & 345 & HM755115 & СMAT03 & CMITS039 & 48.0 & 445 & HM755302 & CMITS035 \\
\hline & CM2939 & СMAT08 & 95.9 & 341 & HM755116 & CMAT01 & CMITS001 & 47.7 & 445 & HM755303 & CMITS001 \\
\hline & CM2940 & CMAT02 & 95.9 & 343 & HM755117 & CMAT01 & CMITS040 & 47.4 & 445 & HM755304 & CMITS036 \\
\hline & CM2941 & CMAT01 & 96.0 & 344 & HM755118 & CMAT01 & CMITS041 & 47.7 & 445 & HM755305 & CMITS037 \\
\hline & CM2942 & CMAT04 & 95.9 & 345 & HM755119 & CMAT01 & CMITS042 & 47.4 & 445 & HM755306 & CMITS038 \\
\hline & CM2943 & CMAT01 & 96.0 & 344 & HM755120 & CMAT01 & CMITS043 & 47.9 & 445 & HM755307 & CMITS039 \\
\hline & CM2944 & СМАТ01 & 96.0 & 344 & HM755121 & СMAT01 & CMITS044 & 48.1 & 445 & HM755308 & CMITS040 \\
\hline & CM2945 & CMAT26 & 95.7 & 344 & HM755122 & CMAT17 & CMITS045 & 47.7 & 445 & HM755309 & CMITS041 \\
\hline \multirow{7}{*}{$\begin{array}{l}\text { 5. Xiaogan, } \\
\text { Hubei Province, } \\
\text { China } \\
(15 ; 2009.07 .14)\end{array}$} & CM2949 & СМАТ07 & 95.9 & 339 & HM755123 & СMAT01 & CMITS002 & 47.9 & 445 & HM755310 & CMITS002 \\
\hline & CM2950 & CMAT03 & 95.9 & 342 & HM755124 & CMAT01 & CMITS046 & 47.4 & 447 & HM755311 & CMITS042 \\
\hline & CM2951 & СМАТ02 & 95.9 & 343 & HM755125 & СMAT01 & CMITS047 & 47.7 & 445 & HM755312 & CMITS043 \\
\hline & CM2952 & CMAT27 & 95.6 & 341 & HM755126 & CMAT18 & CMITS001 & 47.7 & 445 & HM755313 & CMITS001 \\
\hline & CM2953 & CMAT05 & 95.9 & 345 & HM755127 & CMAT01 & CMITS048 & 47.7 & 445 & HM755314 & CMITS044 \\
\hline & CM2954 & CMAT01 & 96.0 & 344 & HM755128 & CMAT01 & CMITS001 & 47.7 & 445 & HM755315 & CMITS001 \\
\hline & CM2955 & СMAT28 & 95.7 & 344 & HM755129 & CMAT04 & CMITS002 & 47.9 & 445 & HM755316 & CMITS002 \\
\hline
\end{tabular}

Continued on next page 
Table 1. Continued.

\begin{tabular}{|c|c|c|c|c|c|c|c|c|c|c|c|}
\hline \multirow{2}{*}{$\begin{array}{l}\text { Collecting locality } \\
\text { (sample size, } \\
\text { collecting date) }\end{array}$} & \multirow{2}{*}{$\begin{array}{l}\text { Animal } \\
\text { number }\end{array}$} & \multicolumn{5}{|c|}{$\mathrm{A}+\mathrm{T}$-rich region } & \multicolumn{5}{|c|}{ ITS2 } \\
\hline & & $\begin{array}{c}\text { Haplotype } \\
\text { I* }^{*}\end{array}$ & $\begin{array}{c}\text { AT } \\
\text { content } \\
(\%) \\
\end{array}$ & $\begin{array}{l}\text { Sequence } \\
\text { size (bp) }\end{array}$ & $\begin{array}{c}\text { GenBank } \\
\text { accession } \\
\text { No. }\end{array}$ & $\begin{array}{c}\text { Haplotype } \\
\text { II }^{* *}\end{array}$ & $\begin{array}{l}\text { Sequence } \\
\text { type I* }\end{array}$ & $\begin{array}{c}\text { GC } \\
\text { content } \\
(\%) \\
\end{array}$ & $\begin{array}{l}\text { Sequence } \\
\text { size } \\
\text { (bp) }\end{array}$ & $\begin{array}{c}\text { GenBank } \\
\text { accession } \\
\text { No. }\end{array}$ & $\begin{array}{l}\text { Sequence } \\
\text { type II** }\end{array}$ \\
\hline \multirow{23}{*}{$\begin{array}{l}\text { 6. Hangzhou, } \\
\text { Zhejiang Province, } \\
\text { China } \\
(15 ; 2009.07 .25)\end{array}$} & CM2956 & CMAT02 & 95.9 & 343 & HM755130 & CMAT01 & CMITS001 & 47.7 & 445 & HM755317 & CMITS001 \\
\hline & CM2957 & CMAT02 & 95.9 & 343 & HM755131 & СMAT01 & CMITS049 & 47.6 & 445 & HM755318 & CMITS045 \\
\hline & CM2958 & СMAT02 & 95.9 & 343 & HM755132 & СMAT01 & CMITS050 & 48.3 & 445 & HM755319 & CMITS046 \\
\hline & CM2959 & CMAT29 & 95.7 & 344 & HM755133 & СMAT05 & CMITS051 & 48.1 & 445 & HM755320 & CMITS047 \\
\hline & CM2960 & СMAТ30 & 95.9 & 342 & HM755134 & CMAT19 & CMITS052 & 47.7 & 447 & HM755321 & CMITS048 \\
\hline & CM2961 & CMAT31 & 95.7 & 344 & HM755135 & CMAT20 & CMITS053 & 47.7 & 447 & HM755322 & CMITS049 \\
\hline & CM2962 & CMAT32 & 95.7 & 344 & HM755136 & CMAT21 & CMITS001 & 47.7 & 445 & HM755323 & CMITS001 \\
\hline & CM2963 & CMAT33 & 95.6 & 342 & HM755137 & CMAT04 & CMITS054 & 48.1 & 445 & HM755324 & CMITS050 \\
\hline & CM2980 & СMAT34 & 95.9 & 341 & HM755138 & CMAT22 & CMITS055 & 47.2 & 447 & HM755325 & CMITS051 \\
\hline & CM2981 & CMAT35 & 95.9 & 345 & HM755139 & CMAT01 & CMITS056 & 47.6 & 445 & HM755326 & CMITS052 \\
\hline & CM2982 & CMAT36 & 95.6 & 345 & HM755140 & СMAT23 & CMITS001 & 47.7 & 445 & HM755327 & CMITS001 \\
\hline & CM2983 & СМАТ37 & 95.7 & 346 & HM755141 & СMAT24 & CMITS057 & 47.2 & 447 & HM755328 & CMITS053 \\
\hline & CM2984 & СMAT02 & 95.9 & 343 & HM755142 & СMAT01 & CMITS004 & 47.7 & 447 & HM755329 & CMITS002 \\
\hline & CM2985 & СMAT38 & 95.7 & 344 & HM755143 & CMAT25 & CMITS001 & 47.7 & 445 & HM755330 & CMITS001 \\
\hline & CM2986 & CMAT02 & 95.9 & 343 & HM755144 & СMAT01 & CMITS058 & 47.9 & 445 & HM755331 & CMITS054 \\
\hline & CM2987 & СMAT39 & 95.9 & 344 & HM755145 & CMAT26 & CMITS059 & 48.1 & 445 & HM755332 & CMITS055 \\
\hline & CM2988 & CMAT40 & 96.0 & 344 & HM755146 & CMAT27 & CMITS060 & 47.4 & 445 & HM755333 & CMITS056 \\
\hline & CM2989 & CMAT41 & 95.9 & 343 & HM755147 & СMAT01 & CMITS061 & 47.4 & 445 & HM755334 & CMITS057 \\
\hline & CM2990 & CMAT01 & 96.0 & 344 & HM755148 & CMAT01 & CMITS001 & 47.7 & 445 & HM755335 & CMITS001 \\
\hline & CM2991 & СMAT08 & 95.9 & 341 & HM755149 & СМАТ01 & CMITS062 & 47.7 & 445 & HM755336 & CMITS058 \\
\hline & CM2992 & CMAT01 & 96.0 & 344 & HM755150 & CMAT01 & CMITS063 & 47.9 & 447 & HM755337 & CMITS059 \\
\hline & CM2993 & СMAT01 & 96.0 & 344 & HM755151 & СMAT01 & CMITS064 & 47.5 & 448 & HM755338 & CMITS060 \\
\hline & CM2994 & CMAT01 & 96.0 & 344 & HM755152 & CMAT01 & CMITS001 & 47.7 & 445 & HM755339 & CMITS001 \\
\hline \multirow{15}{*}{$\begin{array}{l}\text { 7. Naju, } \\
\text { Chonnam } \\
\text { Province, Korea } \\
(15,2008.09 .26)\end{array}$} & CM3001 & CMAT05 & 95.9 & 345 & HM755153 & СMAT01 & CMITS009 & 47.3 & 448 & HM755340 & CMITS001 \\
\hline & CM3002 & СMAT03 & 95.9 & 342 & HM755154 & CMAT01 & CMITS065 & 47.4 & 445 & HM755341 & CMITS061 \\
\hline & CM3003 & CMAT03 & 95.9 & 342 & HM755155 & CMAT01 & CMITS066 & 47.9 & 445 & HM755342 & CMITS062 \\
\hline & CM3004 & CMAT42 & 95.7 & 343 & HM755156 & CMAT28 & CMITS010 & 47.9 & 445 & HM755343 & CMITS006 \\
\hline & CM3005 & CMAT43 & 95.3 & 345 & HM755157 & СМАТ02 & CMITS010 & 47.9 & 445 & HM755344 & CMITS006 \\
\hline & CM3006 & СMAT01 & 96.0 & 344 & HM755158 & СМАТ01 & CMITS003 & 47.7 & 445 & HM755345 & CMITS003 \\
\hline & CM3007 & CMAT02 & 95.9 & 343 & HM755159 & СMAT01 & CMITS067 & 47.4 & 445 & HM755346 & CMITS063 \\
\hline & CM3008 & CMAT01 & 96.0 & 344 & HM755160 & CMAT01 & CMITS068 & 47.9 & 445 & HM755347 & CMITS008 \\
\hline & CM3009 & CMAT44 & 95.3 & 342 & HM755161 & CMAT29 & CMITS069 & 47.7 & 448 & HM755348 & CMITS064 \\
\hline & CM3010 & CMAT45 & 95.9 & 342 & HM755162 & CMAT01 & CMITS070 & 47.6 & 445 & HM755349 & CMITS065 \\
\hline & CM3011 & CMAT01 & 96.0 & 344 & HM755163 & CMAT01 & CMITS001 & 47.7 & 445 & HM755350 & CMITS001 \\
\hline & CM3012 & CMAT07 & 95.9 & 339 & HM755164 & CMAT01 & CMITS001 & 47.7 & 445 & HM755351 & CMITS001 \\
\hline & CM3013 & СMAT01 & 96.0 & 344 & HM755165 & СМАТ01 & CMITS003 & 47.7 & 445 & HM755352 & CMITS003 \\
\hline & CM3014 & CMAT01 & 96.0 & 344 & HM755166 & СМАТ01 & CMITS002 & 47.9 & 445 & HM755353 & CMITS002 \\
\hline & CM3015 & CMAT01 & 96.0 & 344 & HM755167 & СMAT01 & CMITS011 & 47.5 & 447 & HM755354 & CMITS003 \\
\hline \multirow{15}{*}{$\begin{array}{l}\text { 8. Chongju, } \\
\text { Chongbuk } \\
\text { Province, Korea } \\
(15 ; 2009.08 .26)\end{array}$} & CM3020 & CMAT04 & 95.9 & 345 & HM755168 & CMAT01 & CMITS071 & 47.9 & 447 & HM755355 & CMITS066 \\
\hline & CM3021 & CMAT01 & 96.0 & 344 & HM755169 & CMAT01 & CMITS072 & 48.5 & 445 & HM755356 & CMITS067 \\
\hline & CM3022 & СMAT01 & 96.0 & 344 & HM755170 & СMAT01 & CMITS073 & 47.4 & 445 & HM755357 & CMITS068 \\
\hline & CM3023 & CMAT46 & 95.7 & 344 & HM755171 & СМАТ30 & CMITS074 & 47.9 & 447 & HM755358 & CMITS069 \\
\hline & CM3024 & CMAT47 & 96.0 & 344 & HM755172 & СMAТ31 & CMITS075 & 47.5 & 446 & HM755359 & CMITS003 \\
\hline & CM3025 & CMAT48 & 95.6 & 348 & HM755173 & СMAT01 & CMITS007 & 47.3 & 448 & HM755360 & CMITS003 \\
\hline & CM3026 & CMAT49 & 95.7 & 344 & HM755174 & CMAT32 & CMITS004 & 47.7 & 447 & HM755361 & CMITS002 \\
\hline & CM3027 & CMAT50 & 95.9 & 345 & HM755175 & СMAТ33 & CMITS076 & 47.2 & 447 & HM755362 & CMIT070 \\
\hline & CM3028 & CMAT01 & 96.0 & 344 & HM755176 & CMAT01 & CMITS077 & 47.3 & 448 & HM755363 & CMITS071 \\
\hline & CM3029 & CMAT51 & 95.6 & 343 & HM755177 & СMAT04 & CMITS006 & 47.8 & 445 & HM755364 & CMITS005 \\
\hline & CM3030 & СMAT01 & 96.0 & 344 & HM755178 & СMAT01 & CMITS078 & 47.7 & 447 & HM755365 & CMITS072 \\
\hline & CM3031 & CMAT01 & 96.0 & 344 & HM755179 & CMAT01 & CMITS079 & 47.4 & 445 & HM755366 & CMIT073 \\
\hline & CM3032 & CMAT52 & 95.7 & 343 & HM755180 & СMAТ34 & CMITS003 & 47.7 & 445 & HM755367 & CMITS003 \\
\hline & CM3033 & CMAT53 & 95.9 & 343 & HM755181 & CMAT35 & CMITS080 & 47.9 & 447 & HM755368 & CMITS074 \\
\hline & CM3034 & CMAT54 & 95.7 & 343 & HM755182 & СMAT36 & CMITS081 & 47.9 & 444 & HM755369 & CMITS075 \\
\hline \multirow{13}{*}{$\begin{array}{l}\text { 9. Andong, } \\
\text { Kyongbuk } \\
\text { Province, Korea } \\
(15 ; 2009.09 .05)\end{array}$} & CM3127 & СMAT03 & 95.9 & 342 & HM755183 & СMAT01 & CMITS082 & 47.9 & 445 & HM755370 & CMITS076 \\
\hline & CM3128 & CMAT01 & 96.0 & 344 & HM755184 & CMAT01 & CMITS083 & 48.1 & 447 & HM755371 & CMITS077 \\
\hline & CM3129 & CMAT55 & 95.9 & 342 & HM755185 & CMAT01 & CMITS004 & 47.7 & 447 & HM755372 & CMITS002 \\
\hline & CM3130 & CMAT56 & 96.0 & 344 & HM755186 & СМАТ37 & CMITS084 & 47.9 & 445 & HM755373 & CMITS078 \\
\hline & CM3131 & СMAT04 & 95.9 & 345 & HM755187 & СMAT01 & CMITS012 & 47.9 & 445 & HM755374 & CMITS007 \\
\hline & CM3132 & CMAT01 & 96.0 & 344 & HM755188 & СMAT01 & CMITS085 & 48.5 & 445 & HM755375 & CMITS079 \\
\hline & CM3133 & CMAT09 & 96.0 & 346 & HM755189 & CMAT01 & CMITS086 & 48.1 & 445 & HM755376 & CMITS080 \\
\hline & CM3134 & CMAT04 & 95.9 & 345 & HM755190 & СMAT01 & CMITS087 & 47.8 & 445 & HM755377 & CMITS081 \\
\hline & CM3135 & СMAT01 & 96.0 & 344 & HM755191 & СMAT01 & CMITS001 & 47.7 & 445 & HM755378 & CMITS001 \\
\hline & CM3136 & CMAT57 & 95.9 & 340 & HM755192 & CMAT01 & CMITS001 & 47.7 & 445 & HM755379 & CMITS001 \\
\hline & CM3137 & CMAT58 & 95.6 & 341 & HM755193 & CMAT38 & CMITS088 & 47.4 & 447 & HM755380 & CMITS082 \\
\hline & CM3138 & CMAT01 & 96.0 & 344 & HM755194 & CMAT01 & CMITS001 & 47.7 & 445 & HM755381 & CMITS001 \\
\hline & CM3139 & CMAT01 & 96.0 & 344 & HM755195 & СМАТ01 & CMITS089 & 47.5 & 447 & HM755382 & CMITS083 \\
\hline
\end{tabular}

Continued on next page 
Table 1. Continued.

\begin{tabular}{|c|c|c|c|c|c|c|c|c|c|c|c|}
\hline \multirow{2}{*}{$\begin{array}{l}\text { Collecting locality } \\
\text { (sample size, } \\
\text { collecting date) }\end{array}$} & \multirow{2}{*}{$\begin{array}{l}\text { Animal } \\
\text { number }\end{array}$} & \multicolumn{5}{|c|}{$\mathrm{A}+\mathrm{T}$-rich region } & \multicolumn{5}{|c|}{ ITS2 } \\
\hline & & $\begin{array}{c}\text { Haplotype } \\
\text { I* }^{*}\end{array}$ & $\begin{array}{c}\mathrm{AT} \\
\text { content } \\
(\%)\end{array}$ & $\begin{array}{l}\text { Sequence } \\
\text { size (bp) }\end{array}$ & $\begin{array}{c}\text { GenBank } \\
\text { accession } \\
\text { No. }\end{array}$ & $\begin{array}{c}\text { Haplotype } \\
\text { II** }\end{array}$ & $\begin{array}{l}\text { Sequence } \\
\text { type I* }\end{array}$ & $\begin{array}{c}\mathrm{GC} \\
\text { content } \\
(\%) \\
\end{array}$ & $\begin{array}{l}\text { Sequence } \\
\text { size } \\
\text { (bp) }\end{array}$ & $\begin{array}{c}\text { GenBank } \\
\text { accession } \\
\text { No. }\end{array}$ & $\begin{array}{l}\text { Sequence } \\
\text { type II** }\end{array}$ \\
\hline \multirow{17}{*}{$\begin{array}{l}\text { 10. Yesan, } \\
\text { Chungnam } \\
\text { Province, Korea } \\
(15 ; 2009.08 .29)\end{array}$} & CM3140 & CMAT10 & 94.0 & 341 & HM755196 & СMAT01 & CMITS090 & 47.5 & 447 & HM755383 & CMITS084 \\
\hline & CM3141 & CMAT59 & 96.0 & 344 & HM755197 & СМАТ39 & CMITS001 & 47.7 & 445 & HM755384 & CMITS001 \\
\hline & CM3090 & СMAT04 & 95.9 & 345 & HM755198 & СМАТ01 & CMITS091 & 47.2 & 447 & HM755385 & CMITS085 \\
\hline & CM3091 & CMAT06 & 95.9 & 342 & HM755199 & СMAT01 & CMITS001 & 47.7 & 445 & HM755386 & CMITS001 \\
\hline & CM3092 & СMAT60 & 95.7 & 344 & HM755200 & СМАТ02 & CMITS092 & 48.1 & 445 & HM755387 & CMITS086 \\
\hline & CM3093 & CMAT61 & 95.7 & 344 & HM755201 & CMAT40 & CMITS093 & 48.3 & 445 & HM755388 & CMITS087 \\
\hline & CM3094 & СMAT01 & 96.0 & 344 & HM755202 & СMAT01 & CMITS094 & 47.9 & 444 & HM755389 & CMITS008 \\
\hline & CM3095 & СMAT03 & 95.9 & 342 & HM755203 & СMAT01 & CMITS001 & 47.7 & 445 & HM755390 & CMITS001 \\
\hline & CM3096 & CMAT01 & 96.0 & 344 & HM755204 & CMAT01 & CMITS095 & 48.3 & 445 & HM755391 & CMITS088 \\
\hline & CM3097 & СMAT62 & 95.6 & 341 & HM755205 & CMAT02 & CMITS003 & 47.7 & 445 & HM755392 & CMITS003 \\
\hline & CM3098 & СMAT01 & 96.0 & 344 & HM755206 & CMAT01 & CMITS007 & 47.3 & 448 & HM755393 & CMITS003 \\
\hline & CM3099 & CMAT63 & 95.7 & 344 & HM755207 & CMAT41 & CMITS001 & 47.7 & 445 & HM755394 & CMITS001 \\
\hline & CM3100 & CMAT64 & 95.9 & 343 & HM755208 & СMAT01 & CMITS096 & 48.1 & 445 & HM755395 & CMITS089 \\
\hline & CM3101 & CMAT65 & 95.9 & 345 & HM755209 & CMAT01 & CMITS097 & 47.8 & 445 & HM755396 & CMITS090 \\
\hline & CM3102 & CMAT66 & 95.9 & 345 & HM755210 & CMAT42 & CMITS098 & 48.1 & 445 & HM755397 & CMITS091 \\
\hline & CM3103 & СMAT01 & 96.0 & 344 & HM755211 & СМАТ01 & CMITS099 & 47.7 & 447 & HM755398 & CMITS092 \\
\hline & CM3104 & СMAT01 & 96.0 & 344 & HM755212 & CMAT01 & CMITS008 & 47.5 & 447 & HM755399 & CMITS001 \\
\hline \multirow{15}{*}{$\begin{array}{l}\text { 11. Geosan, } \\
\text { Chungbuk } \\
\text { Province, Korea } \\
(15 ; 2009.08 .27)\end{array}$} & CM3042 & CMAT67 & 95.3 & 340 & HM755213 & CMAT43 & CMITS100 & 47.9 & 445 & HM755400 & CMITS093 \\
\hline & CM3043 & CMAT06 & 95.9 & 342 & HM755214 & CMAT01 & CMITS101 & 48.1 & 445 & HM755401 & CMITS094 \\
\hline & CM3044 & CMAT68 & 95.3 & 341 & HM755215 & СМАТ03 & CMITS001 & 47.7 & 445 & HM755402 & CMITS001 \\
\hline & CM3045 & СMAT01 & 96.0 & 344 & HM755216 & СМАT01 & CMITS003 & 47.7 & 445 & HM755403 & CMITS003 \\
\hline & CM3046 & СMAT01 & 96.0 & 344 & HM755217 & СMAT01 & CMITS102 & 47.3 & 444 & HM755404 & CMITS095 \\
\hline & CM3047 & СМАТ09 & 96.0 & 346 & HM755218 & СMAT01 & CMITS001 & 47.7 & 445 & HM755405 & CMITS001 \\
\hline & CM3048 & CMAT69 & 95.6 & 343 & HM755219 & CMAT44 & CMITS001 & 47.7 & 445 & HM755406 & CMITS001 \\
\hline & CM3049 & CMAT10 & 95.9 & 341 & HM755220 & CMAT01 & CMITS103 & 47.7 & 445 & HM755407 & CMITS096 \\
\hline & CM3050 & CMAT70 & 95.6 & 343 & HM755221 & CMAT45 & CMITS104 & 47.4 & 445 & HM755408 & CMITS097 \\
\hline & CM3051 & CMAT71 & 95.9 & 345 & HM755222 & CMAT46 & CMITS001 & 47.7 & 445 & HM755409 & CMITS001 \\
\hline & CM3052 & CMAT72 & 95.9 & 342 & HM755223 & CMAT47 & CMITS105 & 47.9 & 445 & HM755410 & CMITS098 \\
\hline & CM3053 & СMAT06 & 95.9 & 342 & HM755224 & СМАТ01 & CMITS106 & 47.9 & 447 & HM755411 & CMITS002 \\
\hline & CM3054 & CMAT73 & 95.1 & 343 & HM755225 & CMAT48 & CMITS005 & 48.1 & 445 & HM755412 & CMITS005 \\
\hline & CM3055 & CMAT74 & 95.6 & 342 & HM755226 & СMAT02 & CMITS107 & 48.0 & 448 & HM755413 & CMITS099 \\
\hline & CM3056 & CMAT75 & 95.6 & 343 & HM755227 & СMAT02 & CMITS108 & 47.4 & 445 & HM755414 & CMITS100 \\
\hline \multirow{15}{*}{$\begin{array}{l}\text { 12. Suwon, } \\
\text { Kyonggi } \\
\text { Province, Korea } \\
(15 ; 2009.08 .28)\end{array}$} & CM3069 & CMAT76 & 95.9 & 340 & HM755228 & СMAT01 & CMITS001 & 47.7 & 445 & HM755415 & CMITS001 \\
\hline & CM3070 & СMAT09 & 96.0 & 346 & HM755229 & СMAT01 & CMITS109 & 47.7 & 448 & HM755416 & CMITS 101 \\
\hline & CM3071 & СMAT01 & 96.0 & 344 & HM755230 & CMAT01 & CMITS110 & 47.7 & 444 & HM755417 & CMITS102 \\
\hline & CM3072 & CMAT77 & 95.7 & 347 & HM755231 & CMAT05 & CMITS111 & 47.6 & 445 & HM755418 & CMITS 103 \\
\hline & CM3073 & СМАТ09 & 96.0 & 346 & HM755232 & СMAT01 & CMITS112 & 47.4 & 445 & HM755419 & CMITS104 \\
\hline & CM3074 & СMAT06 & 95.9 & 342 & HM755233 & СMAT01 & CMITS113 & 47.7 & 445 & HM755420 & CMITS105 \\
\hline & CM3075 & СMAT02 & 95.9 & 343 & HM755234 & СMAT01 & CMITS114 & 47.9 & 445 & HM755421 & CMITS106 \\
\hline & CM3076 & СМАТ02 & 95.9 & 343 & HM755235 & СMAT01 & CMITS115 & 47.6 & 445 & HM755422 & CMITS107 \\
\hline & CM3077 & CMAT78 & 95.7 & 344 & HM755236 & CMAT49 & CMITS001 & 47.7 & 445 & HM755423 & CMITS001 \\
\hline & CM3078 & СМАТ04 & 95.9 & 345 & HM755237 & СMAT01 & CMITS001 & 47.7 & 445 & HM755424 & CMITS001 \\
\hline & CM3079 & СMAT09 & 96.0 & 346 & HM755238 & CMAT01 & CMITS116 & 47.9 & 445 & HM755425 & CMITS108 \\
\hline & CM3080 & СМАТ04 & 95.9 & 345 & HM755239 & СMAT01 & CMITS004 & 47.7 & 447 & HM755426 & CMITS002 \\
\hline & CM3081 & CMAT79 & 95.9 & 344 & HM755240 & CMAT50 & CMITS117 & 47.5 & 448 & HM755427 & CMITS003 \\
\hline & CM3082 & СMAT80 & 95.9 & 341 & HM755241 & СМАТ01 & CMITS118 & 47.7 & 445 & HM755428 & CMITS109 \\
\hline & CM3083 & CMAT81 & 95.9 & 341 & HM755242 & СMAT01 & CMITS119 & 47.9 & 445 & HM755429 & CMITS 110 \\
\hline \multirow{15}{*}{$\begin{array}{l}\text { 13. Kangreung, } \\
\text { Kangwon } \\
\text { Province, Korea } \\
(15 ; 2009.09 .05)\end{array}$} & CM3150 & СMAT01 & 96.0 & 344 & HM755243 & CMAT01 & CMITS002 & 47.9 & 445 & HM755430 & CMITS002 \\
\hline & CM3151 & CMAT82 & 95.7 & 344 & HM755244 & CMAT51 & CMITS007 & 47.3 & 448 & HM755431 & CMITS003 \\
\hline & CM3152 & CMAT83 & 95.7 & 344 & HM755245 & CMAT52 & CMITS120 & 47.7 & 447 & HM755432 & CMITS111 \\
\hline & CM3153 & CMAT01 & 96.0 & 344 & HM755246 & СMAT01 & CMITS121 & 47.5 & 447 & HM755433 & CMITS003 \\
\hline & CM3154 & СMAT84 & 95.7 & 344 & HM755247 & CMAT53 & CMITS122 & 47.1 & 448 & HM755434 & CMITS112 \\
\hline & CM3155 & CMAT85 & 95.6 & 345 & HM755248 & CMAT54 & CMITS123 & 47.9 & 445 & HM755435 & CMITS113 \\
\hline & CM3156 & СMAT86 & 95.7 & 343 & HM755249 & CMAT55 & CMITS124 & 47.9 & 445 & HM755436 & CMITS114 \\
\hline & CM3157 & CMAT87 & 95.6 & 342 & HM755250 & CMAT56 & CMITS012 & 47.9 & 445 & HM755437 & CMITS007 \\
\hline & CM3158 & CMAT88 & 95.3 & 340 & HM755251 & CMAT57 & CMITS011 & 47.5 & 447 & HM755438 & CMITS003 \\
\hline & CM3159 & СMAT89 & 95.9 & 343 & HM755252 & CMAT58 & CMITS125 & 47.5 & 448 & HM755439 & CMITS115 \\
\hline & CM3160 & СMAT90 & 95.9 & 343 & HM755253 & CMAT01 & CMITS001 & 47.7 & 445 & HM755440 & CMITS001 \\
\hline & CM3161 & CMAT91 & 95.7 & 344 & HM755254 & CMAT59 & CMITS126 & 47.7 & 445 & HM755441 & CMITS116 \\
\hline & CM3162 & CMAT92 & 95.6 & 344 & HM755255 & CMAT60 & CMITS127 & 47.4 & 445 & HM755442 & CMITS117 \\
\hline & CM3163 & CMAT93 & 95.7 & 343 & HM755256 & CMAT61 & CMITS128 & 47.2 & 447 & HM755443 & CMITS118 \\
\hline & CM3164 & CMAT94 & 95.7 & 344 & HM755257 & CMAT62 & CMITS129 & 47.1 & 445 & HM755444 & CMITS119 \\
\hline
\end{tabular}

*Haplotype or sequence type I: sequences obtained before the Gblocks analysis (Castresana, 2000). **Haplotype or sequence type II: sequences obtained after the Gblocks analysis (Castresana, 2000), which removed non-conserved blocks in the alignment. 


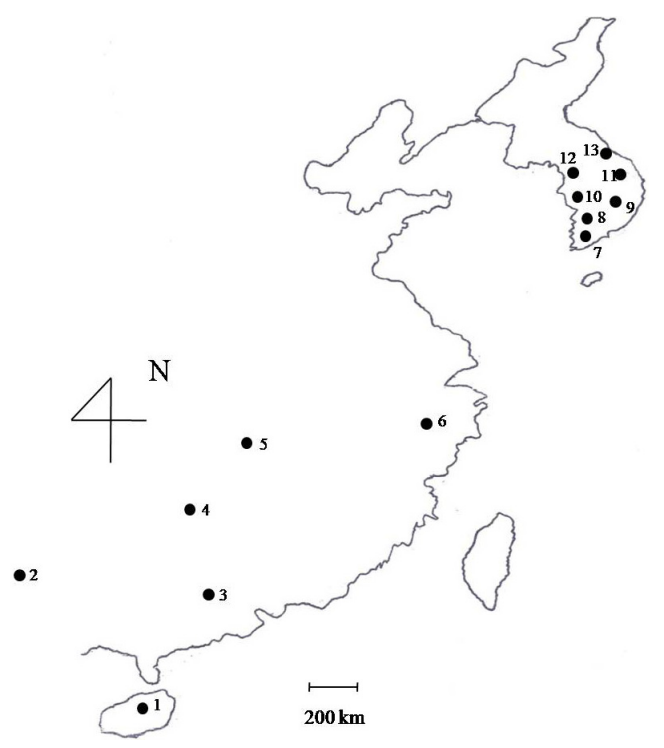

Figure 1. Sampling locations of Cnaphalocrocis medinalis in Korea and China. General locality names are as follows: 1. Haikou, Hainan Province, China; 2. Tonghai, Yunnan Province, China; 3. Guangzhou, Guangdong Province, China; 4. Changsha, Hunan Province, China; 5. Xiaogan, Hubei Province, China; 6. Hangzhou, Zhejiang Province, China; 7. Naju, Chonnam Province, Korea; 8. Chongju, Chongbuk Province, Korea; 9. Andong, Kyongbuk Province, Korea; 10. Yesan, Chungnam Province, Korea; 11. Geosan, Chungbuk Province, Korea; 12. Suwon, Kyonggi Province, Korea; 13. Kangreung, Kangwon Province, Korea.

\section{Sequence analysis}

The entire A+T-rich region was delimited by aligning the sequences with the fulllength mitogenome sequences of lepidopterans (Yukuhiro et al., 2002; Cameron and Whiting, 2008), whereas the entire ITS 2 was delimited via HMM-based ITS 2 annotation (Keller et al., 2009). All A+T-rich region and ITS2 sequence alignments were conducted using MAFFT ver. 6 (Katoh et al., 2002), with the gap opening penalty set to 1.53 and the offset value ( $\approx$ gap extension penalty) set to 0.5 . When the homologous sequences from two individuals differed by $\geq 1$ nucleotide base or one insertion/deletion (indel) position, the sequences were regarded as different haplotypes (the A+T-rich region) or sequence types (ITS2). Haplotype or sequence type designations were applied to new sequences as they were discovered (i.e., CMAT01, CMAT02, and so on for the A+T-rich region; CMITS001, CMITS002, and so on for ITS2). Finally, 94 haplotypes from the A+T-rich region and 129 sequence types from the ITS2 region were acquired and deposited in the GenBank database under the accession Nos. HM755071-HM755257 for the A+T-rich region and HM755258-HM755444 for ITS2, respectively (Table 1).

In order to more accurately align the A+T-rich and ITS2 region sequences for the subsequent phylogenetic and population level analyses, the Gblocks (ver. 0.91b) software (Castresana, 2000) was employed for the selection of conserved regions. The alignment option, "allow the smaller final blocks", was used for both the A+T-rich region and ITS2; as a result, the following parameters were used: minimum number of sequences for a conserved position $=45(\mathrm{~A}+\mathrm{T}-\mathrm{rich}$ region) and 65 (ITS2), minimum number of sequences for a flanking position $=74$ (A+T-rich 
region) and 109 (ITS2), maximum number of contiguous nonconserved positions $=8$, minimum length of a block $=5$, and gap positions $=$ zero. After the Gblocks analysis, all indels were eventually removed. Finally, 62 new A+T-rich region haplotypes with 312 bp from 94 haplotypes and 119 new ITS2 sequence types with 439 bp from 129 ITS2 sequences were obtained.

\section{Phylogenetic analysis}

To determine the relationships among the $62 \mathrm{~A}+\mathrm{T}$-rich region haplotypes and 119 ITS2 sequence types acquired from the 187 individuals of $C$. medinalis and to detect any groups, phylogenetic analysis was conducted using the PAUP ver. 4.0b10 software (Swofford, 2002) via the maximum-parsimony method (Fitch, 1971), using a heuristic search strategy starting with stepwise addition trees replicated 100 times, and a random input order of sequences to generate the initial tree for each replicate. The robustness of maximum-parsimony topologies was assessed via bootstrapping with 1000 replicates (tree-bisection-reconnection heuristic search) of 100 random stepwise addition replicates each, for all analyses. The A+Trich region and ITS2 of the within-family species Locastra muscosalis and Teliphasa elegans were utilized as outgroups to root the trees.

\section{Estimates of population characteristics}

Haplotype diversity and nucleotide diversity, both of which are reflective of genetic diversity within each locality, were determined using the Arlequin ver. 3.0 software (Excoffier et al., 2005). Maximum sequence divergence within each locality was estimated via extraction of the within-locality estimates of unrooted pairwise distances in PAUP ver. 4.0b (Swofford, 2002).

The genetic distance was estimated using the Arlequin ver. 3.0 software (Excoffier et al., 2005). Population pairwise genetic distance $\left(F_{\mathrm{ST}}\right)$ and a permutation test of the significant differentiation of the pairs of localities (1000 bootstraps) were evaluated in accordance with the approach described by Excoffier et al. (1992), wherein the distance between DNA sequences was calculated via the Tamura method (Tamura, 1992), which is an extension of the Kimura 2-parameters method (Kimura, 1980), allowing for unequal nucleotide frequencies. This option computes transition-transversion ratios, as well as the overall nucleotide frequencies from the original data. Pairwise $N_{\mathrm{m}}$ (the product of the effective population size, $N_{\mathrm{e}}$, and migration rate, $\mathrm{m})$ values were employed to estimate the pairwise $F_{\mathrm{ST}}$ based on the equilibrium relationship: $F_{\mathrm{ST}}=1 /\left(2 N_{\mathrm{m}}+1\right)(1)$ for the A+T-rich region and $F_{\mathrm{ST}}=1 /\left(4 N_{\mathrm{m}}+1\right)(2)$ for ITS 2.

In order to determine whether any isolation-by-distance (IBD) effect has occurred in C. medinalis populations, matrices of genetic distance data $\left[F_{\mathrm{ST}} /\left(1-F_{\mathrm{ST}}\right)\right]$ and the logarithms of geographical distance data $(\mathrm{ln} \mathrm{km})$ among the Chinese population and Korean population sampling sites were respectively constructed. These matrices were then analyzed to determine their degree of correlation via a Mantel test, with significance tests conducted over 10,000 randomizations (Mantel, 1967). The analysis was conducted using the isolation-by-distance software package, with the negative genetic distance set to zero (Bohonak, 2002).

\section{Hierarchical analysis}

In order to determine the degree of hierarchical subdivision between specified sets of lo- 
calities (i.e., Chinese localities and Korean localities), analysis of molecular variance (AMOVA) was also performed using Arlequin ver. 3.0 (Excoffier et al., 2005). A hierarchical analysis of variance was conducted to partition total variance into variance components attributable to interpopulation differences. Variance components were then employed to compute the fixation indices, and their significance was assessed by 1000 permutations, as described by Excoffier et al. (1992).

\section{RESULTS}

\section{Sequence analysis}

Ninety-four mitochondrial A+T-rich region haplotypes (CMAT01-CMAT94), ranging in size from 339 to $348 \mathrm{bp}$, were obtained from 187 C. medinalis specimens. The average A+T content of the 94 haplotypes was $95.6 \%$, with a range of $95.1-96.0 \%$ (Table 1), suggesting very strong $\mathrm{A}+\mathrm{T}$ bias; this is consistent with what has been found for other lepidopterans (Cameron and Whiting, 2008). The sequence alignment of 94 haplotypes revealed 112 variable sites, accounting for $31.82 \%$ of the A+T-rich region sequences; this total includes 33 sites of mono- or dinucleotide insertions/deletions (indels) and 79 nucleotide substitution sites. Uncorrected pairwise distance among 94 haplotypes evidenced a maximum sequence divergence of $4.55 \%$, with 16 variable positions. After the Gblocks analysis, which only selects conserved blocks, $62 \mathrm{~A}+\mathrm{T}-\mathrm{rich}$ region haplotypes were newly obtained, and the length was truncated to $312 \mathrm{bp}$ (Table 1). The sequence alignment of these newly generated haplotypes evidenced 79 nucleotide substitution sites, with no indels. Uncorrected pairwise distance among the new 62 haplotypes evidenced a maximum sequence divergence of $1.60 \%$, with five variable positions. We conducted a preliminary sequencing of the $658 \mathrm{bp}$ of the mitochondrial COI genes corresponding to the "DNA Barcode" region, using the primers LepF (5'-ATTCAACCAATCATAAAGATATTGG-3') and LepR (5'-TAAACTTCTGGATGTCCAAAAAATCA-3') (Hajibabaei et al., 2006) from 20 samples collected from each Korean (Naju, locality 7) and Chinese population (Xiaogan, locality 5). However, all sequence analyses of the samples revealed identical sequences. Thus, the A+T-rich region of $C$. medinalis exhibited substantially higher sequence variation than the COI gene.

In the case of ITS2, 129 sequence types were obtained via the sequencing of ITS2 rDNA from the 187 C. medinalis specimens (Table 1). The 129 ITS2 sequences of C. medinalis ranged in size from 444 to $450 \mathrm{bp}$, and they harbored an average $\mathrm{G}+\mathrm{C}$ content of $47.8 \%$, with a range from 47.1 to $48.5 \%$. Sequence alignment revealed 165 variable sites, consisting of 10 sites of mono- or dinucleotide indels and 155 sites of nucleotide substitutions. Uncorrected pairwise distance among 129 sequence types (CMITS001-CMITS129) evidenced a maximum divergence of $4.43 \%$, with 20 variable sites. Following the Gblocks analysis, the sequence length and the number of sequence types were reduced to $439 \mathrm{bp}$ and 119 , respectively, with a maximum sequence divergence of $3.19 \%$, containing 14 variable positions.

\section{Analysis of A+T-rich region structure}

Substantial efforts have been made to understand the role of the A+T-rich region, but only limited success has been achieved thus far. Some of the progress made to date has involved the A+T-rich region for the replication origin for both mtDNA strands in Drosophila species (Fauron and Wolstenholme, 1980; Saito et al., 2005) and for minor-strand mtDNA 
only in a few insect species, including the lepidopteran Bombyx mori (Saito et al., 2005). Other sources of information were consulted to look for conserved sequences associated with the regulatory role of the A+T-rich region; such signals are known to be engraved on the nucleotide sequences of the region, in the form of structural elements. These have included descriptions of the sequence region from the orthopterans Schistocerca gregaria and Chorthippus parallelus (Zhang et al., 1995), and partially from the lepidopteran Parnassius bremeri (Kim et al., 2009). Therefore, we searched such conserved sequences using a representative of the C. medinalis haplotype (CMAT01) that was detected in all populations, along with two additional haplotypes (Figure 2). The very obvious structural element detected was the poly-T stretch, which was expected to be present at the 5'-end (upstream of srRNA) of the A+T-rich region (Saito et al., 2005). This poly-T stretch has been suggested to be composed of a minimum of 10 thymidine nucleotides, to function as a possible recognition site for the initiation of replication of the major-strand mtDNA (Saito et al., 2005). In the haplotype CMAT01, one poly-T stretch of $13 \mathrm{bp}$ was detected in the beginning region, starting at nucleotide position 28, as 5'-TTTTTTTTTTTTT-3' (Figure 2). In other haplotypes, the lengths of the poly-T stretches range from 10-16 bp. Besides the poly-T stretch, a 4-bp long conserved motif sequence, 5'-ATAG-3', was detected at nucleotide position 24 (Figure 2). This sequence has been detected in the A+T-rich region of all sequenced lepidopterans, but in most cases, the 5-bp long motif sequence 5'-ATAGA-3' was identified. This motif has also been suggested to perform some regulatory role together with the poly-T stretch (Kim et al., 2009).

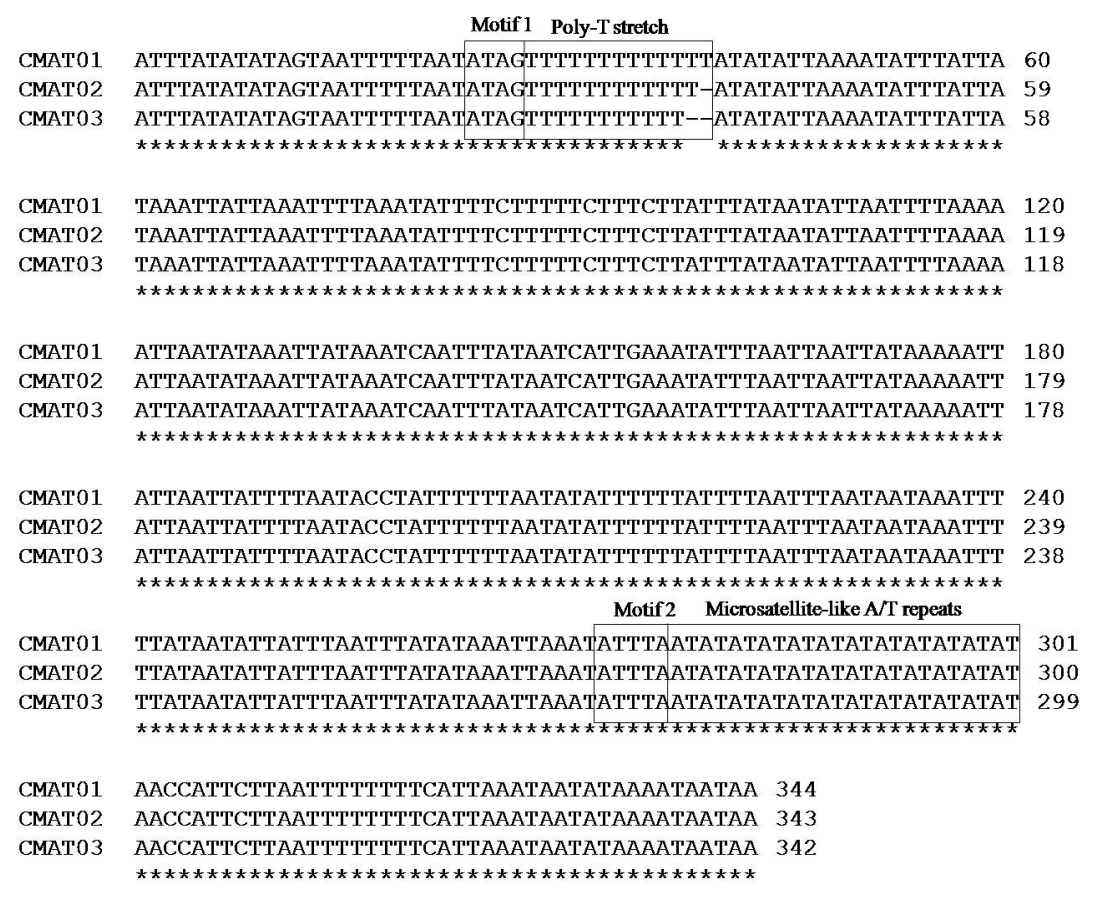

Figure 2. Sequence alignment of three haplotypes of the Cnaphalocrocis medinalis A+T-rich region. Asterisks indicate identical nucleotides among the three haplotypes. One stretch of the poly-T, microsatellite-like AT repeats, and two conserved motif sequences are indicated by boxes in the sequence. The sequences are listed in the $5^{\prime}$ to $3^{\prime}$ direction. 
Another element, referred to as the microsatellite-like AT repeat stretch, which was supposed to be preceded by the ATTTA motif detected in most other sequenced lepidopteran A+T-rich regions (Cameron and Whiting, 2008), was apparent in the C. medinalis $\mathrm{A}+\mathrm{T}-$-rich region. One region harbored such stretches, which differed in length and core repeats among haplotypes, but was composed principally of the (AT) $)_{n}$ sequence (Figure 2 ). Such tandem repetition in the A+T-rich region has been suggested to be the relevant site of the origin of DNA replication and the formation of the stem-and-loop structure, which is important in the initiation of replication (Zhang et al., 1995).

\section{Phylogenetic analysis}

In order to evaluate the relevant phylogenetic relationships and to detect any discernable groups in connection with geographic distribution, phylogenetic analysis was conducted via the maximum-parsimony method. The analysis based on the $62 \mathrm{~A}+\mathrm{T}-$ rich region haplotypes revealed a major body of unresolved haplotypes, but only a few haplotypes were clustered in the four phylogenetic groups that were weakly supported by bootstrap analysis $(<65 \%$, Figure 2$)$. Each of these groups was composed of a maximum of two haplotypes originating from different localities, respectively. For example, one group that was composed of haplotypes CMAT11 and CMAT47 was corroborated by a bootstrap support of $65 \%$, and these haplotypes originated from localities 2 and 11, respectively (Figure 3), which were separated by a linear stretch of at least $2800 \mathrm{~km}$ (Figure 1). A similar clustering pattern can also be detected in other groups. Thus, the overall results of phylogenetic analysis indicate a random association of haplotypes, independent of geographic origin, possibly implying substantial gene flow in this species.

According to the phylogenetic results obtained from the ITS2 sequence types, only one discernable group (61\%) was detected among the 119 analyzed sequence types (Figure 4). This group was composed of two sequence types obtained from localities 2 and 9 , which are also quite distant from one another (Figure 4). Overall, the phylogenetic results obtained from the ITS2 sequence types were quite similar to those obtained from the A+T-rich region.

\section{Genetic diversity, genetic distance, and gene flow}

Within-locality diversity was estimated in terms of haplotype or sequence type diversity $(\mathrm{H})$, maximum sequence divergence, mean number of pairwise differences, and nucleotide diversity $(\pi)$ (Table 2 ). With regard to the $\mathrm{H}$ values, the highest estimate in the $\mathrm{A}+\mathrm{T}$-rich region was observed in Kangreung (locality 13) at 0.9714, whereas in ITS2, the H values in Haikou (locality 1), Changsha (locality 4), Chongju (locality 8), Suwon (locality 12), and Kangreung (locality 13) were the same as detected in the A+T-rich regions. The lowest $\mathrm{H}$ in the A+T-rich region was detected in the Guangzhou (locality 3 ) population as 0.0000 , whereas that of ITS2 was detected in the Tonghai population (locality 2 ) as 0.8000 . In terms of $\pi$, the lowest and highest estimates in the A+T-rich region were detected in Guangzhou (locality 3) and Kangreung (locality 13) as 0.0000 and 0.0073 , respectively. In ITS2, however, they were found in Hangzhou (locality 6) and Xiaogan (locality 5), at 0.0054 and 0.0097 , respectively. These results demonstrate that the populations with the extreme estimates frequently evidence substantial differences between the two molecules. This may reflect population dynamics related to the organism's behavior, population history, or different evolutionary modes of each molecule. 
62 CMAT28 (locality 7$)$

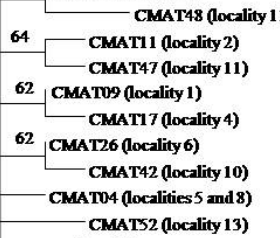

CMAT01 (localities $1,2,3,4,5,6,7,8,9,10,11,12$ and 13)

CMAT1 4 (locality 4)

CMAT25 (locality 6)

CMAT02 (localities 7, 10 and 11 )

CMAT03 (localities 2, 4 and 11)

CMATI0 (locality 1)

CMAT12 (locality 2)

CMAT13 (locality 2)

CMATI5 (locality 4)

CMAT30 (locality 8)

CMAT40 (locality 10)

CMAT1 6 (locality 4)

CMAT29 (locality 7$)$

CMATS3 (locality 13)

CMAT62 (locality 13)

CMAT23 (locality 6)

CMAT37 (locality9)

CMAT19 (locality 5)

CMAT18 (locality 5)

100

CMAT21 (locality5)

CMATS5 (locality 13)

CMATzo (locality 5)

CMAT39 (locality9)

CMAT46 (locality 11)

CMAT38 (locality 9)

CMAT32 (locality 8)

CMAT45 (locality 11)

CMAT61 (locality 13)

CMATS4 (locality 13)

CMAT43 (locality 11)

CMAT22 (ocality 6)

CMATS7 (locality 13)

CMAT27 (locality 6)

CMAT59 (locality 13)

CMAT34 (locality 8)

CMATS6 (locality 13)

CMAT36 (locality 8)

CMAT35 (locality 8)

CMATS8 (locality 13)

CMAT08 (locality 1)

CMAT60 (locality 13)

CMAT41 (locality 10)

CMAT06 (locality 1)

CMAT33 (locality 8)

CMAT24 (locality 6)

CMATO7 (locality 1)

CMATOS (localities 5 and 12)

CMAT31 (locality 8)

CMAT44 (locality 11)

CMATSO (locality 12)

CMATS1 (locality 12)

Teliphasa eleguow

Figure 3. Phylogenetic analysis of 62 haplotypes of the Cnaphalocrocis medinalis A+T-rich region. The tree was acquired via the MP method incorporated in the PAUP ver. $4.0 \mathrm{~b} 10$ software (Swofford, 2002). The tree length is 178 steps, the consistency index is 0.949 , and retention index is 0.839 . Locastra muscosalis and Teliphasa elegans belonging to the same family were incorporated in the analysis in order to root the tree. The numbers on the branches represent bootstrap values of 1000 replications. 


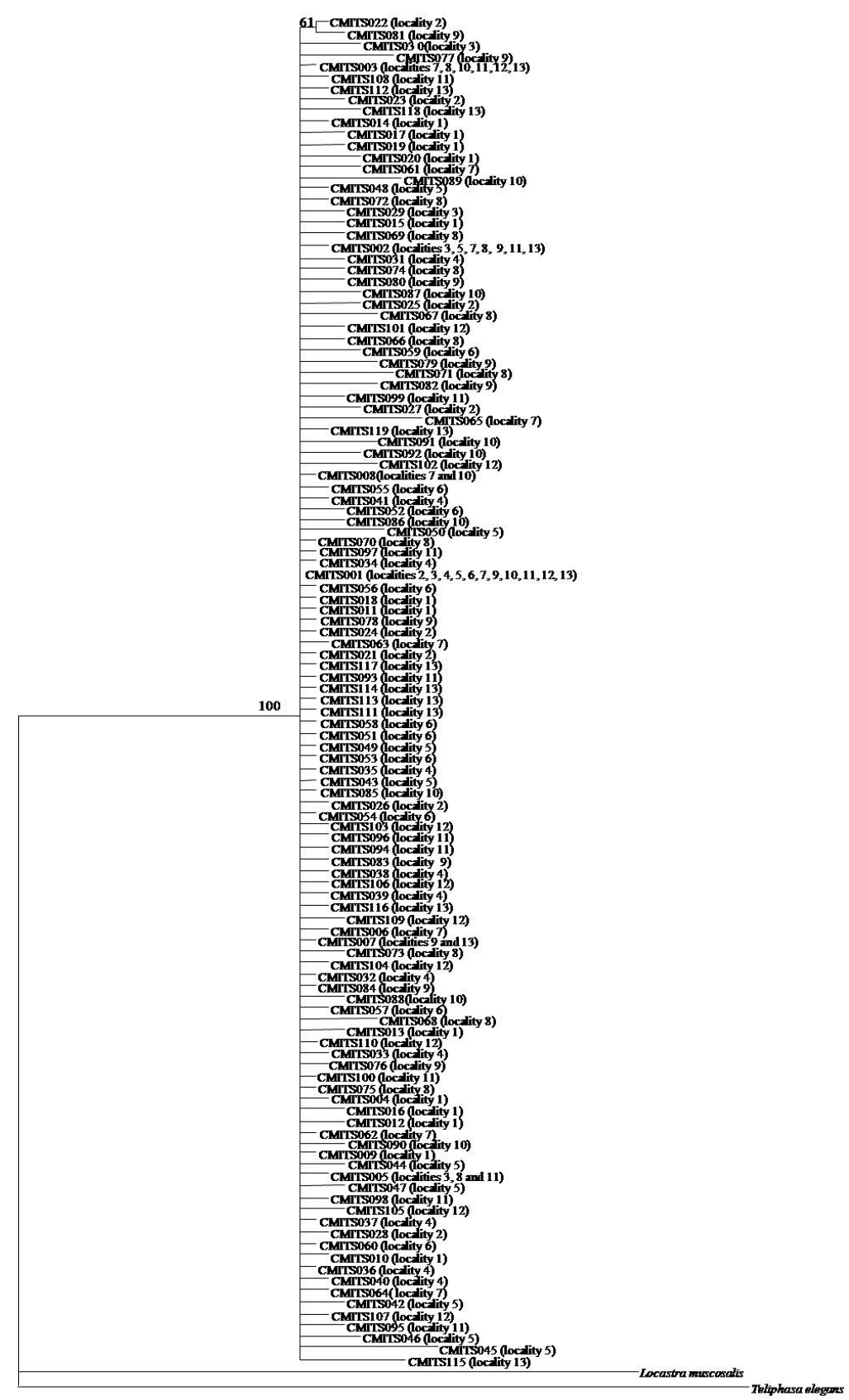

Figure 4. Phylogenetic analysis of 119 sequence types of Cnaphalocrocis medinalis ITS2. The tree was acquired via the MP method incorporated in the PAUP ver. $4.0 \mathrm{~b} 10$ software (Swofford, 2002). The tree length is 533 steps, the consistency index is 0.689 , and the retention index is 0.311 . Locastra muscosalis and Teliphasa elegans belonging to the same family were incorporated in the analysis in order to root the tree. The numbers on the branches represent bootstrap values of 1000 replications. 


\begin{tabular}{|c|c|c|c|c|c|c|c|c|c|c|c|c|c|}
\hline & \multirow[t]{2}{*}{ SS } & \multicolumn{2}{|c|}{$\mathrm{NH}$} & \multicolumn{2}{|c|}{$\mathrm{H}$} & \multicolumn{2}{|c|}{ NP } & \multicolumn{2}{|c|}{$\operatorname{MSD}(\%)$} & \multicolumn{2}{|c|}{ MPD } & \multicolumn{2}{|c|}{$\pi$} \\
\hline & & $\mathrm{A}+\mathrm{T}$ & ITS2 & $\mathrm{A}+\mathrm{T}$ & ITS2 & $\mathrm{A}+\mathrm{T}$ & ITS2 & $\mathrm{A}+\mathrm{T}$ & ITS2 & $\mathrm{A}+\mathrm{T}$ & ITS2 & $\mathrm{A}+\mathrm{T}$ & ITS2 \\
\hline 1. Haikou & 15 & 6 & 13 & 0.5714 & 0.9714 & 5 & 19 & 1.4097 & 2.6246 & 0.6667 & 4.0000 & 0.0021 & 0.0091 \\
\hline 2. Tonghai & 15 & 5 & 9 & 0.4762 & 0.8000 & 5 & 15 & 1.3237 & 2.4327 & 0.6667 & 2.4000 & 0.0021 & 0.0055 \\
\hline 3. Guangzhou & 7 & 1 & 4 & 0.0000 & 0.8571 & 0 & 5 & 0.0000 & 1.9909 & 0.0000 & 2.4762 & 0.0000 & 0.0056 \\
\hline 4. Changsha & 15 & 6 & 13 & 0.6476 & 0.9714 & 6 & 18 & 1.7237 & 2.4327 & 0.9143 & 2.8381 & 0.0029 & 0.0065 \\
\hline 5. Xiaogan & 15 & 7 & 11 & 0.7238 & 0.9333 & 6 & 27 & 1.4097 & 3.6815 & 0.9143 & 4.2667 & 0.0029 & 0.0097 \\
\hline 6. Hangzhou & 15 & 7 & 12 & 0.6571 & 0.9429 & 7 & 14 & 1.7237 & 2.2233 & 0.9333 & 2.3905 & 0.0030 & 0.0054 \\
\hline 7. Naju & 15 & 4 & 10 & 0.3714 & 0.9333 & 4 & 17 & 1.7237 & 3.1261 & 0.5333 & 3.1619 & 0.0017 & 0.0072 \\
\hline 8. Chongju & 15 & 9 & 13 & 0.8000 & 0.9714 & 10 & 24 & 1.9871 & 3.1261 & 1.3333 & 3.9238 & 0.0043 & 0.0089 \\
\hline 9. Andong & 15 & 5 & 12 & 0.5619 & 0.9429 & 6 & 25 & 1.9871 & 3.1261 & 0.9143 & 3.9619 & 0.0029 & 0.0090 \\
\hline 10. Yesan & 15 & 4 & 11 & 0.3714 & 0.9333 & 3 & 21 & 1.4097 & 2.8015 & 0.4000 & 3.6381 & 0.0013 & 0.0083 \\
\hline 11. Geosan & 15 & 9 & 12 & 0.8476 & 0.9429 & 12 & 16 & 2.2181 & 2.2233 & 1.7143 & 2.5333 & 0.0055 & 0.0058 \\
\hline 12. Suwon & 15 & 4 & 13 & 0.3714 & 0.9714 & 3 & 19 & 1.4097 & 2.8025 & 0.4000 & 3.4286 & 0.0013 & 0.0078 \\
\hline 13. Kangreung & 15 & 13 & 13 & 0.9714 & 0.9714 & 17 & 17 & 1.9871 & 2.9691 & 2.2667 & 3.0952 & 0.0073 & 0.0071 \\
\hline
\end{tabular}

$\mathrm{SS}=$ sample size; $\mathrm{NH}=$ number of haplotypes; $\mathrm{H}=$ haplotype/sequence type diversity; $\mathrm{NP}=$ number of polymorphic sites; $\mathrm{MSD}=$ maximum sequence divergence; $\mathrm{MPD}=$ mean number of pairwise differences; $\pi=$ nucleotide diversity.

The genetic distances $\left(F_{\mathrm{ST}}\right)$ between pairs of populations in the $\mathrm{A}+\mathrm{T}$-rich region and ITS2 are provided in Tables 3 and 4, respectively. For the A+T-rich region, $F_{\mathrm{ST}}$ ranged from -0.061 (comparison between localities 2 and 3 ), which is effectively zero (due to negative value), to 0.020 (comparisons between localities 4 and 5, between 4 and 10, and between 5 and 10), with a range of migration rate $\left(N_{\mathrm{m}}\right)$ from 24.00 to infinite. No significant genetic differentiation whatsoever was detected $(\mathrm{P}>0.05)$ in 78 comparisons, suggesting a high interrelationship of the C. medinalis populations in terms of the maternally inherited mitochondrial genome (Table 3 ). In the case of ITS2, the $F_{\mathrm{ST}}$ value ranged from - 0.026 (comparison between localities 5 and 12) to 0.082 (comparison between localities 1 and 8), with a range of $N_{\mathrm{m}}$ from 5.601 to infinite. Unlike the $F_{\mathrm{ST}}$ estimates obtained from the A+T-rich region, 13 population pairs evidenced significant differentiation $(\mathrm{P}<0.05)$ among 78 comparisons. Among them, the remote island of Haikou (locality 1), was significantly different from all other populations, except for Guangzhou (locality 3) (Table 4). The two additional population pairs are localities 4 and 8 , and localities 6 and 8 (Table 4). These results indicate genetic subdivision in a few C. medinalis populations, including Haikou (locality 1 ) in terms of the bi-parentally inherited nuclear genome. Nevertheless, those population pairs that evidenced significant $F_{\mathrm{ST}}$ values still had somewhat high estimates of $N_{\mathrm{m}}$, ranging from 5.601 to 11.40 , meaning an exchange of approximately 5 to 11 individual migrants of $C$. medinalis. Overall, the genetic distance and gene flow data indicate that $C$. medinalis populations are connected to one another quite well, but the nuclear ITS2 sequence reveals a somewhat higher degree of genetic differentiation relative to the $\mathrm{A}+\mathrm{T}-$-rich region sequence.

\section{Isolation by distance}

The results of the Mantel test (10,000 randomizations) for the detection of IBD provided an $r$ (correlation coefficient) value of $0.135(\mathrm{P}=0.88)$ and $0.209(\mathrm{P}=0.86)$ in the $\mathrm{A}+\mathrm{T}-$ rich region among Chinese populations and among Korean populations, respectively. On the other hand, the $\mathrm{r}$ value based on ITS2 was $0.001(\mathrm{P}=0.49)$ and $0.192(\mathrm{P}=0.86)$ among the Chinese populations and Korean populations, respectively. These results indicate no IBD effect among the Chinese and Korean populations (Figure 5). 


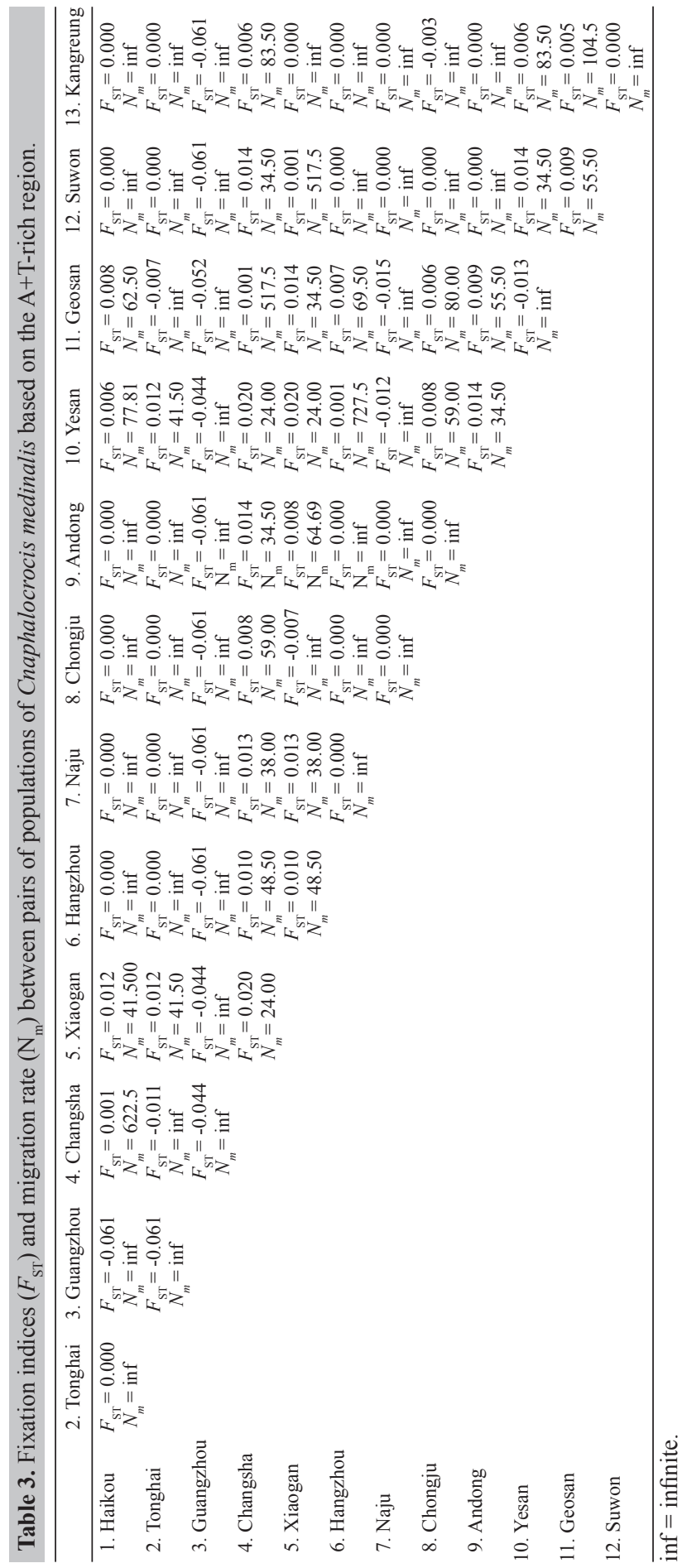




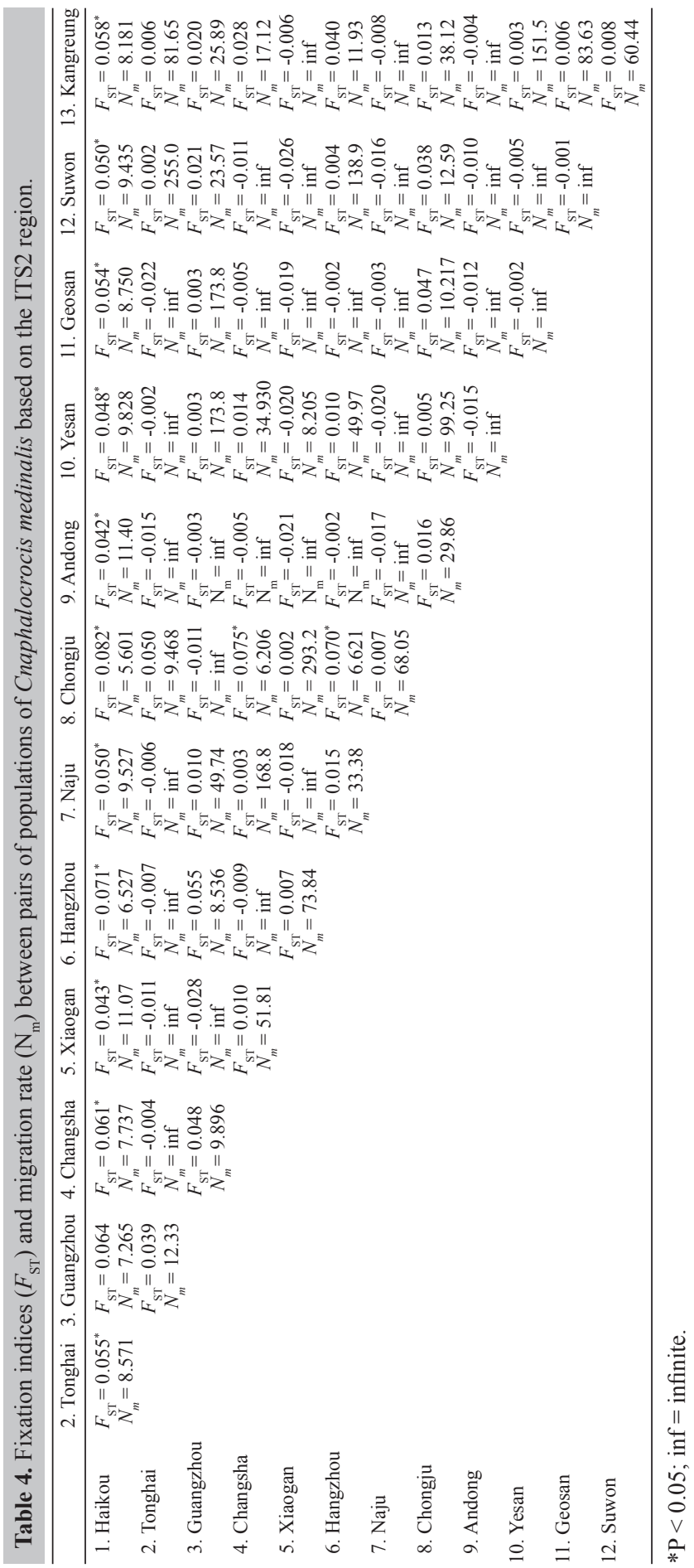



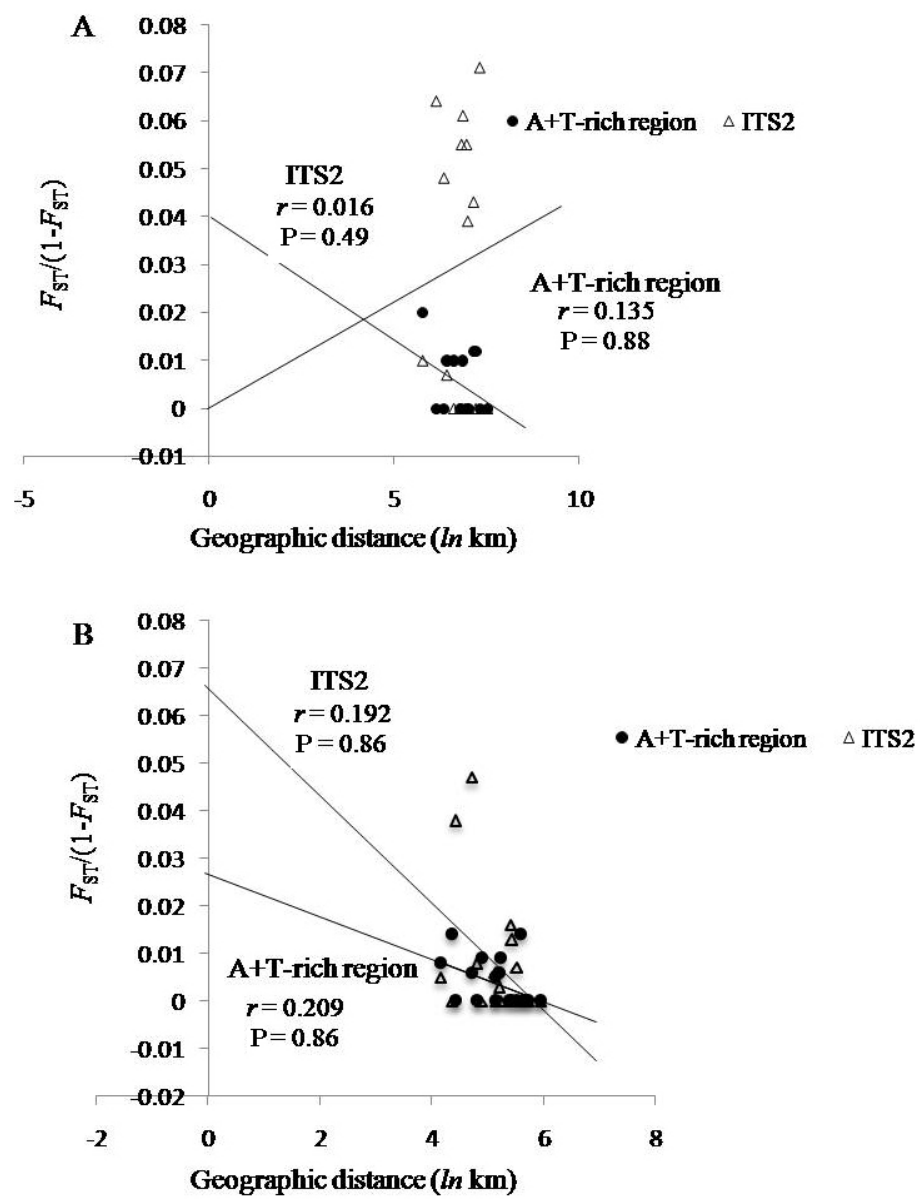

Figure 5. Scatter plots of genetic distance $v s$ geographical distance $(\ln )$ for pairwise Korean and Chinese Cnaphalocrocis medinalis population comparisons based on two molecular markers. A. Isolation by distance among Chinese populations (the $\mathrm{A}+\mathrm{T}$-rich region: $\mathrm{r}=0.135, \mathrm{P}=0.88$; ITS2: $\mathrm{r}=0.001, \mathrm{P}=0.49$ ). $\mathbf{B}$. Isolation by distance among Korean populations (the A+T-rich region: $\mathrm{r}=0.209, \mathrm{P}=0.86$; ITS2: $\mathrm{r}=0.192, \mathrm{P}=0.86$ ).

\section{Population genetic structure}

The hierarchical partition of genetic variance and fixation index of each hierarchical level by AMOVA is shown in Table 5. The analyses based on both the A+T-rich region and ITS2 showed that the majority of variance was present in the within-populations (99.93 and $98.48 \%$, respectively), rather than the among-population within-regions or the among-regions. This indicates that each population of $C$. medinalis is composed of heterogeneous individuals. In ITS2, although it is not particularly high, a significant amount of the among-population variance component $(1.20 \%)$ was detected in the among-population within-regions, and the estimate was significant $(\mathrm{P}<0.005)$ (Table 5). This probably is reflective of a degree of divergence of certain C. medinalis from others, in that some population pairs, including the Haikou 
(locality 1 ) and Chongju (locality 8) pairs, showed significant $F_{\text {ST }}$ estimates in ITS2 (Table 4; Figure 1). On the other hand, the A+T-rich region evidenced no significant variance component of the among-population within-regions (Table 5). This result is consistent with the $F_{\text {ST }}$ estimates obtained from the A+T-rich region (Table 3). Finally, the variance components of the among-regions based on both the A+T-rich region and ITS 2 were only -0.03 and $0.32 \%$, respectively, with no significance (Table 5), thus suggesting absence of a populational genetic structure between China and Korea.

Table 5. Hierarchical analysis based on the A+T-rich region and ITS2 in Cnaphalocrocis medinalis.
\begin{tabular}{llrrrrc}
\hline Sequence & Source of variation & \\
\hline A+T-rich region & Among-regions & d.f. & Sum of squares & $\%$ & \multicolumn{1}{c}{$\Phi$} & P \\
& Among-population within-regions & 1 & 0.508 & 0.09 & 0.00094 & NS \\
& Within-populations & 11 & 5.138 & -0.03 & -0.00028 & NS \\
ITS2 & 174 & 81.600 & 99.93 & 0.00067 & NS \\
& Among-regions & 1 & 2.413 & 0.32 & 0.00316 & NS \\
& Among-population within-regions & 11 & 21.152 & 1.20 & 0.01202 & $*$ \\
& Within-populations & 174 & 284.895 & 98.48 & 0.01515 & $*$ \\
\hline
\end{tabular}

${ }^{a}$ The scheme for among-region was established by subdividing six Chinese localities (localities 1, 2, 3, 4, 5, and 6) as one group and seven Korean localities (localities 7, 8, 9, 10,11, 12, and 13) as another group; d.f. = degrees of freedom; $\%=$ percentage of variation; $\Phi=$ fixation indices; $\mathrm{P}=$ significance of percentage of variation and fixation indices estimated from permutation tests (1000 permutations) (Excoffier et al., 1992). NS = not significant; ${ }^{*} \mathrm{P}<0.05$.

\section{DISCUSSION}

\section{Genetic diversity}

For the rice leaf roller, C. medinalis, the leaf part of rice can be considered a major resource for both egg-laying and larval feeding. During their generations they run rampant, damaging rice leaves; the reduction of output has been reported in a range between $20-60 \%$ in China (Riley et al., 1995). Their dispersal capacity is also quite well known; insect monitoring radar has shown that $C$. medinalis can migrate in large numbers (Riley et al., 1995). Thus, the relatively high dispersal capacity, coupled with the abundance of rice fields in China and Korea, allow us to expect a high genetic diversity in C. medinalis. In fact, the pairwise comparisons between pairs of populations obtained both from the A+T-rich region and ITS2 sequences evidenced an overall relatively high estimate of $N_{\mathrm{m}}$, primarily without any statistically significant $F_{\mathrm{ST}}$, although one Chinese population, the Haikou population (locality 1), evidences a significantly high $F_{\mathrm{ST}}$ compared to most other populations, according to the ITS2 data only (Tables 3 and 4). Furthermore, the results obtained in the AMOVA test indicate that the majority of genetic variance was within-populations, rather than at any other hierarchical level (99.93\% in the A+T-rich region and $98.48 \%$ in the ITS2 sequence, respectively; Table 5), showing that each population of C. medinalis is composed of genetically heterogeneous individuals, as a consequence of high dispersal and the characteristics of large populations.

\section{Genetic similarity among distant populations}

Our $F_{\mathrm{ST}}$ data from both the A+T-rich region and the ITS2 sequences showed almost no significant genetic distance between pairs of populations, even the Chinese and Korean localities (Tables 3 and 4). The results demonstrate substantially high gene flow among the 
C. medinalis populations; this was confirmed by the results of phylogenetic analysis, which showed a random association of haplotypes, regardless of geographic origin (Figures 3 and 4) and IBD, which was not statistically supported (Figure 5).

These results were consistent with field observations. Cnaphalocrocis medinalis can breed all year round in the tropical rice-growing areas, corresponding to locations south of approximately $22^{\circ} \mathrm{N}$ latitude (Zhang et al., 1981). These source populations migrate to southwestern China, and subsequently expand into other parts of China in a northeastern direction, and subsequently into the southern part of the Korean peninsula (Zhang et al., 1981). In fact, several lines of evidence obtained from direct observation support the abundance of moths migrating long distances. For example, Zhang et al. (1981) determined, via a mark-and-recapture method, that C. medinalis migrates for over $800-1100 \mathrm{~km}$ in a northeastward direction in China. Additionally, it has also been determined that $C$. medinalis migrates from China to Japan every year during June-July, with assistance from winds (Miyahara et al., 1981). Similar observations have also been reported regarding migration from southwest China to the southwestern part of the Korean peninsula (Kim and Choi, 1984). Once arriving in Korea during June-July, $C$. medinalis spends two or three generations inhabiting sites in Korea (Kim and Choi, 1984). Considering that the cold temperatures of Korean winter do not allow for C. medinalis to overwinter (Kim and Choi, 1984), populations newly migrated from China are considered to be the source population. Thus, our data in collaboration with the information collected in the field support the supposition of this study, namely that $C$. medinalis possesses sufficient flight capacity for dispersal, resulting in a substantial amount of gene flow among populations and an absence of a populational genetic structure, even between China and Korea.

\section{Genetic differentiation of the Haikou population only by ITS2}

Our $F_{\mathrm{ST}}$ data generated both from the A+T-rich region and the ITS2 sequences showed almost no significant genetic distance between pairs of populations. Exceptions to this observation were seen only with the sequence analysis of ITS2 (Table 4), wherein comparisons of the Haikou population (locality 1) to all other populations, except for Guangzhou (locality 3 ), were made (Figure 1). This may imply that the C. medinalis individuals collected from Haikou (locality 1) were divergent from other populations; this finding requires some explanation. From a geographical perspective, Haikou is located on an offshore island, Hainan Island, which is located $\sim 29.5 \mathrm{~km}$ south of the southern coast of mainland China (Figure 1). It has been determined that the majority of $C$. medinalis occurring in southwestern China including Hainan Island, where Haikou (locality 1) is located, is composed of emigrants dispersed from the source of the tropical rice-growing areas (Zhang et al., 1981). These southwestern Chinese populations are then expanded into China in a northeastern direction and subsequently into the southern part of the Korean peninsula (Zhang et al., 1981). In the case of Haikou (locality 1), where breeding is possible year-round (Chen and Guo, 1996), the pre-existing local subpopulations mingle with the newly dispersed immigrants from June to July for the first time and from August to September for the second time (Chen and Guo, 1996). Because our sample collection in Haikou (locality 1) was conducted on August 15, 2009, which is within the infestation period for both pre-existing populations that have bred independently and newly arrived populations that originated from the tropical rice-growing areas, the population genetic characteristics of the Hainan Island including Haikou population (locality 1) would represent 
a mixture of the populations. Thus, the genetic differentiation of the Haikou population (locality 1) from most other Chinese populations, obtained via ITS2 sequence analysis (Table 4), appears to be the consequence of such a phenomenon.

However, the detection of the genetic differences between the Haikou population (locality 1) and most other populations only by ITS2 sequence analysis may require further explanation. Theoretically, the maternally inherited mtDNA would have an effective population size approximately one-quarter the size of the effective population size based on nuclear makers that are inherited bi-parentally, if the sex ratio is equal (Birky et al., 1983). This has led some to observe that the effects of genetic drift could be substantially stronger in the mtDNA, resulting in more rapid fixation and more profound population subdivision (Birky et al., 1983). However, dispersal functions as a counterforce against genetic drift (Hanski, 1996). In situations in which dispersal is biased to a single gender, the typical expectation of population structure would be falsified (Birky et al., 1983).

The observation of a somewhat higher degree of genetic differentiation in the ITS2 (Table 4) and the detection of significant genetic differentiation of the Haikou population (locality 1) only by bi-parentally inherited ITS2, rather than mtDNA sequence analysis, appears to indicate that female dispersal is more important than male dispersal. No previous studies have found evidence for gender-biased dispersal of $C$. medinalis, with the exception of some studies on the dispersal capacity of each gender within-superfamilial species. For example, Shirai (1998) has demonstrated a longer and continuous flight of the female of the European corn borer, Ostrinia nubilalis (family Crambidae), during the pre-ovipositional period, which lasts three to four days after emergence. Although their analysis did not involve within-familial species, Tu et al. (2010) also demonstrated that the total flight duration and distance of 1-day-old female Spodoptera litura moths during a 72-h period were substantially lengthened, by $24 \mathrm{~h}$ and $105.4 \mathrm{~km}$; this suggests more effective dispersal of females than males. Although it may not be direct evidence, this circumstantial information still implies the importance of female-biased dispersal, considering that insect migration is closely associated with flight ability (Drake and Gatehouse, 1995).

Nevertheless, lack of support for significant $F_{\mathrm{ST}}$ estimates in most population pairs in ITS2 at the same time may also indicate that female-biased dispersal would be relevant only under temporary conditions such as the beginning of infestation, during which it is important to find further abundant host plants for egg-laying and feeding. Instead, overall high withinpopulation genetic diversity, very low variance components of the among-population withinregions and the among-regions, lack of statistical support for isolation by distance, and no significant female-biased dispersal in other population pairs in both mitochondrial and nuclear DNA collectively indicate that migratory C. medinalis populations are well connected to one another by high gene flow, but do not necessarily reveal gender-biased dispersal.

\section{ACKNOWLEDGMENTS}

Research supported by a grant from the Agenda Program (\#200901FHT020609413) from the Rural Development Administration, Republic of Korea.

\section{REFERENCES}

Amos W and Harwood J (1998). Factors affecting levels of genetic diversity in natural populations. Philos. Trans. R. Soc. Lond. B Biol. Sci. 353: 177-186.

Ballard JW and Whitlock MC (2004). The incomplete natural history of mitochondria. Mol. Ecol. 13: 729-744. 
Birky CW Jr, Maruyama T and Fuerst P (1983). An approach to population and evolutionary genetic theory for genes in mitochondria and chloroplasts, and some results. Genetics 103: 513-527.

Bohonak AJ (2002). IBD (isolation by distance): a program for analyses of isolation by distance. J. Hered. 93: 153-154.

Bravo JP, Felipes J, Zanatta DB, Silva JLC, et al. (2008). Sequence and analysis of the mitochondrial DNA control region in the sugarcane borer Diatraea saccharalis (Lepidoptera: Crambidae). Braz. Arch. Biol. Technol. 51: 471-477.

Cameron SL and Whiting MF (2008). The complete mitochondrial genome of the tobacco hornworm, Manduca sexta, (Insecta: Lepidoptera: Sphingidae), and an examination of mitochondrial gene variability within butterflies and moths. Gene 408: 112-123.

Castresana J (2000). Selection of conserved blocks from multiple alignments for their use in phylogenetic analysis. Mol. Biol. Evol. 17: 540-552.

Chen W and Guo Y (1996). Observation on the migrant phenomenon of Cnaphalocrocis medinalis Guenee. Nat. Sci. J. Hainan Univ. 14: 37-42.

Denno RE, Roderick GK, Peterson MA, Huberty AE, et al. (1996). Habitat persistence underlies intraspecific variation in the dispersal strategies of planthoppers. Ecol. Monogr. 66: 389-408.

Depaquit J, Lienard E, Verzeaux-Griffon A, Ferte H, et al. (2008). Molecular homogeneity in diverse geographical populations of Phlebotomus papatasi (Diptera, Psychodidae) inferred from ND4 mtDNA and ITS2 rDNA epidemiological consequences. Infect. Genet. Evol. 8: 159-170.

Drake VA and Gatehouse AG (1995). Insect Migration: Tracking Resources Through Space and Time. In: Migratory Potential in Insects: Variation in an Uncertain Environment (Gatehouse AG and Zhang XX, eds.). Cambridge University Press, London, 193-229.

Excoffier L, Smouse PE and Quattro JM (1992). Analysis of molecular variance inferred from metric distances among DNA haplotypes: application to human mitochondrial DNA restriction data. Genetics 131: 479-491.

Excoffier L, Laval G and Schneider S (2005). Arlequin (version 3.0): an integrated software package for population genetics data analysis. Evol. Bioinform. Online 1: 47-50.

Fauron CM and Wolstenholme DR (1980). Intraspecific diversity of nucleotide sequences within the adenine + thyminerich region of mitochondrial DNA molecules of Drosophila mauritiana, Drosophila melanogaster and Drosophila simulans. Nucleic Acids Res. 8: 5391-5410.

Fitch WM (1971). Toward defining the course of evolution: minimal change for a specific tree topology. Syst. Zool. 20: 406-416.

Hajibabaei M, Janzen DH, Burns JM, Hallwachs W, et al. (2006). DNA barcodes distinguish species of tropical Lepidoptera. Proc. Natl. Acad. Sci. U. S. A. 103: 968-971.

Hanski I (1996). Metapopulation Ecology. In: Population Dynamics in Ecological Space and Time (Rhodes OEJ, Chesser RK and Smith MH, eds.). Univerity of Chicago Press, Chicago, 13-43.

Ji Y, Zhang D and He L (2003). Evolutionary conservation and versatility of a new set of primers for amplifying the ribosomal internal transcribed spacer regions in insects and other invertebrates. Mol. Ecol. Notes 3: 581-585.

Katoh K, Misawa K, Kuma K and Miyata T (2002). MAFFT: a novel method for rapid multiple sequence alignment based on fast Fourier transform. Nucleic Acids Res. 30: 3059-3066.

Keller A, Schleicher T, Schultz J, Muller T, et al. (2009). 5.8S-28S rRNA interaction and HMM-based ITS2 annotation. Gene 430: 50-57.

Kim KC and Choi CS (1984). Studies on the bionomies and analysis of damage of the rice leaf folder, Cnaphalocrocis medinalis G. in South region of Korea. Rural Dev. Rev. 19: 25-32.

Kim MI, Baek JY, Kim MJ, Jeong HC, et al. (2009). Complete nucleotide sequence and organization of the mitogenome of the red-spotted apollo butterfly, Parnassius bremeri (Lepidoptera: Papilionidae) and comparison with other lepidopteran insects. Mol. Cells 28: 347-363.

Kimura M (1980). A simple method for estimating evolutionary rates of base substitutions through comparative studies of nucleotide sequences. J. Mol. Evol. 16: 111-120.

Kisimoto R (1984). Insect pests of the rice plant in Asia. Prot. Ecol. 7: 83-104.

Mantel N (1967). The detection of disease clustering and a generalized regression approach. Cancer Res. 27: 209-220.

Miyahara Y (1981). Occurrence of the rice leafroller in Japan. Jpn Agric. Res. Q. 15: 100-105.

Miyahara Y, Wada T and Kobayashi M (1981). Appearance of Cnaphalocrocis medinalis Guenee in early planted rice fields in Chikugo. Jpn. J. Appl. Entomol. Zool. 25: 26-32.

Nei M and Feldman MW (1972). Identity of genes by descent within and between populations under mutation and migration pressures. Theor. Popul. Biol. 3: 460-465.

Riley JR, Reynolds DR, Smith AD, Edwards AS, et al. (1995). Observations of the autumn migration of the rice leaf roller Cnaphalocrocis medinalis (Lepidoptera: Pyralidae) and other moths in eastern China. Bull. Entomol. Res. 85: 397-414.

Saito S, Tamura K and Aotsuka T (2005). Replication origin of mitochondrial DNA in insects. Genetics 171: 1695-1705.

Shirai J (1998). Laboratory evaluation of flight ability of the Oriental corn borer, Ostrinia furnacalis (Lepidoptera: 
Pyralidae). Bull. Entomol. Res. 88: 327-333.

Swofford DL (2002). PAUP*. Phylogenetic Analysis Using Parsimony (*and Other Method). Ver. 4.10. Sinauer Associates, Sunderland.

Tamura K (1992). Estimation of the number of nucleotide substitutions when there are strong transition-transversion and G+C-content biases. Mol. Biol. Evol. 9: 678-687.

Tu YG, Wu KM, Xue FS and Lu YH (2010). Laboratory evaluation of flight activity of the common cutworm, Spodoptera litura (Lepidoptera: Noctuidae). Insect Sci. 17: 53-59.

Wada T, Ogawa Y and Nakasuga T (1988). Geographical difference in mated status and autumn migration in the rice leaf roller moth, Cnaphalocrocis medinalis. Entomol. Exp. Appl. 46: 141-148.

Yukuhiro K, Sezutsu H, Itoh M, Shimizu K, et al. (2002). Significant levels of sequence divergence and gene rearrangements have occurred between the mitochondrial genomes of the wild mulberry silkmoth, Bombyx mandarina, and its close relative, the domesticated silkmoth, Bombyx mori. Mol. Biol. Evol. 19: 1385-1389.

Zhang DX, Szymura JM and Hewitt GM (1995). Evolution and structural conservation of the control region of insect mitochondrial DNA. J. Mol. Evol. 40: 382-391.

Zhang JF, Kimatu JN, Guo WL and Liu B (2009). Habitat fragmentation causes rapid genetic differentiation and homogenization in natural plant populations - a case study in Leymus chinensis. Afr. J. Biotechnol. 8: 3440-3447.

Zhang XX, Geng JG and Zhou WJ (1981). Studies of the migration patterns of rice leaf roller, Cnaphalocrocis medinalis Guenee in China. J. Nanjing Agric. Coll. 3: 1-12. 